\title{
The benefits of organic farming to spontaneous vascular flora biodiversity, West Pomerania, Poland
}

\author{
Janina Borysiak* \\ Department of Integrated Geography, Adam Mickiewicz University in Poznań, Dzięgielowa 27, 61-680 Poznań, Poland
}

\begin{abstract}
Research was carried out on the importance of organic farming practices for maintaining agricultural landscape complexity with consequent benefits for spontaneous vascular flora biodiversity. An agricultural landscape unit (75 ha) composed of extensively used arable lands and grasslands and small remnant natural habitats, occurring among fields or in field verges in the West Pomerania region, Poland, was investigated. Spontaneous vascular flora of extensively farmed landscape was mapped using the topographic method. The examined flora was analyzed in terms of plant species richness and diversity. The following attributes of flora were considered: taxonomic and syntaxonomic diversity, and the share of geographical and geographical-historical elements, Raunkiaer's life forms, archaeophytes, kenophytes, plants with conservation status and threatened in the Polish regions or countries of the European Union, and ancient woodland plant species indicators. Spontaneous vascular flora included 338 species / 75 ha and represented rich taxonomic diversity: 213 genera, 71 families and 48 orders. The phytocoenoses included 52 plant associations from 17 classes, 23 orders, and 32 alliances of the phytosociological system, including 6 segetal synanthropic communities.
\end{abstract}

Keywords: agroecology; biodiversity; organic farming; spontaneous vascular flora; weediness

\section{Introduction}

In 1989 a market economy, including natural capital, was introduced in Poland. In consequence, changes in land use and land cover have been observed in the whole country [1]. They transform the functions of geoecosystems [2] and the agrarian structure of land $[3,4]$. For instance, this is apparent in West Pomerania where large areas of low production ex-arable crop fields of state farms have been changed into mowed fallows, while in the regions with more fertile soils - agricultural production has been intensified [5]. This has led to an increase in natural plant diversity, like in the case of newly formed grasslands, or loss in plant diversity as a result of agricultural intensification [6]. Due to the intensification of agriculture, serious biodiversity loss has been reported from Great Britain during the last 50 years [7]. In the European Union, the common agricultural policy was established to halt biodiversity loss on farmlands [8]. It is aimed at reducing agricultural intensification through the implementation of multiple function agriculture. One of tools that serves this purpose are agri-environment schemes which in the years 2014-2020 become upgraded to agri-environment-climatic schemes [9]. They implement

\footnotetext{
*Email: janina.borysiak@amu.edu.pl
}

Handling Editor: Elżbieta Weryszko-Chmielewska
EU regulations on natural environment protection, the EU Biodiversity Strategy to 2020 [10], and the EU Landscape Convention [11]. These documents obligate their signatories to identify areas of valuable resources and, then, to protect them. They also inspire scientists to pursue more deeply the ecological and landscape directions of biodiversity studies [12]. The need for multiple function farmlands was indicated in the EU Biodiversity Strategy to 2020. Their creation is based on the knowledge about natural resources not only within a farm, but also in its neighborhood. Also, insufficient effectiveness of agri-environment schemes was emphasized [13], because in case of small fragmented areas of environmental resources this program may not protect some important parts of the target habitat or species population located outside a participating farm. The creation of traditional protected area forms, with groups of farmlands managed using extensive farming methods, is recommended as a more effective tool in attaining the scheme objectives. In Poland the first plots for monitoring natural effects of agri-environment schemes were established not earlier than 2011 [14].

In the years 2013-2014, there were conducted the studies of spontaneous vascular flora in the landscape with arable lands, on which extensive agricultural practices were performed. Additionally, uncultivated field margins and small remnant natural habitats, usually eliminated in case of conventional agriculture, were preserved in this landscape 
used by individual farmers. In the Agri-EnvironmentClimate Scheme 2014-2020 such elements of landscape are treated as ecological focus areas and protected as a part of greening infrastructure, due to their high biodiversity. The aim of the present work was to show the richness and diversity of spontaneous vascular flora of this extensively used agricultural landscape.

\section{Material and methods}

\section{Study area}

An extensively farmed agricultural landscape of 75 ha, situated beside an individual farm in Pustkowie, was selected for the study. Fields were fertilized with animal manure only and were not sprayed with any chemical pesticides. No mechanical weed control methods were used. Crop diversification was used. Potatoes and rye were cultivated. Non-cropped small midfield habitats were maintained. The borders of the research plot were marked out in the field by the most northward - N 5346 $18.56^{\prime \prime} /$ E 16 $29^{\prime} 51.03^{\prime \prime}$, southward -

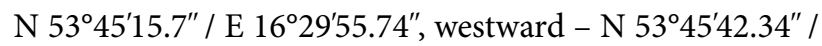
$\mathrm{E} 16^{\circ} 29.35 .62^{\prime \prime}$ and eastward - N 53 ${ }^{\circ} 45^{\prime} 34.54^{\prime \prime} / \mathrm{E} 16^{\circ} 30^{\prime} 17.35^{\prime \prime}$ points. Almost the whole area is situated in the ATPOL square BB-56, while its small northern part in BB-46.

According to physical and geographical regionalization [15], Pustkowie is located in the Drawsko Lakeland mesoregion (314.45), West Pomerania Lakeland macroregion (314.4) and South Baltic Lakeland subprovince (314). It is a lowland area of the early glacial phase of Vistulian glaciation [16], with late glacial and Holocene alterations. The lie of the land and a mosaic arrangement of the Quaternary formations exert a large influence on vegetation landscape. Together with weather conditions of the temperate climate zone, they shape the direction and rate of geo-succession processes [2]. According to the climatic division of Poland [17], the analyzed area is located in the Central Pomerania Region, at an altitude of 107-156 m a.s.l. The surface geological formations are mainly Pleistocene: kame sands, in some places gravels and boulder clays, as well as gravel and dust glacial sands on boulder clay and sands with aqua-glacial gravels. Boulder clays are very sandy, decalcified and strongly weathered, with a high content of erratics in the upper parts [18-20]. The soil cover is mainly made of acidic brown soil, most often developed from poorly clayish sands [21]. In the north-west and south-east regions, the valleys of watercourses are situated. They channel water to the Parsęta river (directly or indirectly). The Pomeranian watercourses are fed by groundwater, rain and snow [22]. The structural elements of the Pustkowie landscape unit studied were as follows: arable lands (66\% of the studied area), an orchard in sod (17\%), meadows (2\%), pasture (2\%), fallows (4\%), tree stands and thickets (4\%), 11 ponds and swamps (4\%), network of balks, watercourses $(1.9 \mathrm{~km})$, dirt roads $(2 \mathrm{~km})$, and farm buildings (1\%).

\section{Field mapping and data analysis}

The object of the study was to assess plant diversity of spontaneous vascular flora, because flora is considered to be a geocomponent of landscape that significantly affects the biodiversity of higher trophic levels [14]. During the vegetation seasons in the years 2013-2014, the flora of Pustkowie was mapped using the topographic method [23], on the basis of a 1:10 000 topographic map and orthomap. Floristic investigations were carried out in spring and summer in all structural elements of the Pustkowie rural landscape, such as arable lands and balks, fallows, orchard, meadows, pasture, tree stands, thickets, ponds and watercourses, swamps, dirt roads, and others.

Ecophysiographic conditions were analyzed using, among others, the following 1:50 000 maps: detailed geological maps with geomorphological features as well as hydrographic, hydrogeological and sozological maps. Some cartographic materials were obtained from the websites http://natura2000. gdos.gov.pl and http://www.geoportal.gov.pl. The geographical coordinates are presented in the WGS 84 system. At the site of each floristic inventory, a plant community of the basic rank was diagnosed in the syntaxonomic system, following the Braun-Blanquet phytosociological method [24]. The obtained result was used, among others, for the determination of natural habitats of the European Ecological Network Natura 2000. A handbook published by the Ministry of Environment [25] and the regulation of the Minister of Environment were used for identification [26]. The results of the phytosociological study will be reported in a separate paper. The examined vascular flora was analyzed in terms of plant species richness and plant diversity. The following aspects were considered: taxonomic position [27], geographic element [28], geographical-historical status [29], contribution of archaeophytes [30] and kenophytes $[31,32]$, syntaxonomic rank $[24,33]$, Raunkiaer's life form [34], conservation status in Poland [35], category of threat in Wielkopolska and West Pomerania [36,37] and in countries of the European Union [38], crop wild relatives at risk of decline in countries of the European Union [38], and ancient woodland plant species indicators $[39,40]$.

\section{Results}

The spontaneous vascular flora of Pustkowie comprised 338 species (Tab. 1). They represented 213 genera, 71 families, and 48 orders. Ten species of Pteridophyta (5 Equisetales and 5 Polypodiales) and 3 Pinopsida (Spermathophyta) were recorded. The largest group of Spermathophyta (325 spp., $96 \%$ of total flora) was Magnoliophytina (254 Magnoliopsida, 71 Liliopsida). The sequence of genera richest in species comprised: Carex (10), Veronica (10), Poa (6), Polygonum (6) and Trifolium (6), while for families: Asteraceae (51 species), Poaceae (42), Rosaceae (19), and Lamiaceae (17). In the Raunkiaer's life form spectrum, hemicryptophytes (191 species, $57 \%)$, terophytes $(93,28 \%)$, fanerophytes (41, $12 \%)$, and geophytes $(39,12 \%)$ prevailed. If a given plant had more than one life form, each of them was included in the calculations. The natural flora consisted of 287 (85\%) spontaneophytes. Among them, 46 (16\%) ancient forest plant species were recorded, while 22 of them were characteristic for Querco-Fagetea. The group of spontaneophytes was composed of three geographic elements: connective (144 species, $50 \%)$, Holarctic $(135,47 \%)$, and cosmopolitan $(8,3 \%)$. The 
Tab. 1 Spontaneous vascular flora of the Pustkowie agricultural landscape (West Pomerania, Poland).

\begin{tabular}{|c|c|c|c|c|c|c|c|c|c|}
\hline 1 & 2 & 3 & 4 & 5 & 6 & 7 & 8 & 9 & 10 \\
\hline Acer platanoides & M & $\mathrm{CE}-\mathrm{M}(\mathrm{m})$ & $\mathrm{sp}$ & QF & QF & *9180 & . & . & . \\
\hline Acer pseudoplatanus & M & $\mathrm{CE}-\mathrm{M}(\mathrm{m})$ & $\mathrm{sp}$ & QF & QF & *9180 & . & . & . \\
\hline Achillea millefolium & $\mathrm{H}$ & sa-ES(w)-M(n)-IR(m) & $\mathrm{sp}$ & MA & MA, TG, A, S & . & . & . & . \\
\hline Achillea ptarmica & $\mathrm{H}$ & sa-ES(w, n) & $\mathrm{sp}$ & MA & A, MA & 6410 & . & . & . \\
\hline Acorus calamus & Hy & Asia C \& S & $\mathrm{kn}$ & $\mathrm{Ph}$ & $\mathrm{Ph}$ & . & . & . & . \\
\hline Adoxa moschatellina & G & $\mathrm{CB}(\mathrm{d})$ & $\mathrm{sp}$ & QF & QF & $9110,9130,9160$ & an & . & . \\
\hline Aegopodium podagraria & $\mathrm{G}, \mathrm{H}$ & sa-ES(w) & $\mathrm{sp}$ & A & QF, A & . & an & . & . \\
\hline Aesculus hippocastanum & M & Eur SE & $\mathrm{kn}$ & - & QF & . & . & . & . \\
\hline Aethusa cynapium & $\mathrm{T}$ & sa-CE & $\mathrm{sp}$ & A & A & . & . & . & . \\
\hline Agrimonia eupatoria & $\mathrm{H}$ & sa-CE-M-IR & $\mathrm{sp}$ & TG & MA, TG & . & . & . & . \\
\hline Agrostis canina & $\mathrm{H}$ & sa-ES & $\mathrm{sp}$ & SC & MA & 7140 & . & $\mathrm{LC}$ & . \\
\hline Agrostis capillaris & $\mathrm{H}$ & sa-ES-M(n) & $\mathrm{sp}$ & $\mathrm{CU}$ & A, MA, TG & 4030 & . & . & . \\
\hline Agrostis gigantea & $\mathrm{H}$ & $\mathrm{CB}$ & $\mathrm{sp}$ & MA & A, MA & . & . & . & . \\
\hline Agrostis stolonifera & $\mathrm{H}$ & $\mathrm{CB}$ & $\mathrm{sp}$ & MA & $\mathrm{B}, \mathrm{MA}, \mathrm{Ph}, \mathrm{S}$ & . & . & $\mathrm{LC}$ & CWR \\
\hline Ajuga reptans & $\mathrm{H}$ & sa-CE-M(n) & $\mathrm{sp}$ & QF & QF & . & an & . & . \\
\hline Alchemilla monticola & $\mathrm{H}$ & $\mathrm{CE}$ & $\mathrm{sp}$ & MA & MA & 6510 & . & . & . \\
\hline Alisma plantago-aquatica & Hy & $\mathrm{CB}$ & $\mathrm{sp}$ & $\mathrm{Ph}$ & $\mathrm{Ph}$ & . & . & $\mathrm{LC}$ & . \\
\hline Alnus glutinosa & M & sa-ES(e)-M(n) & $\mathrm{sp}$ & $\mathrm{Ag}$ & $\mathrm{A}, \mathrm{Ag}$ & . & . & . & . \\
\hline Alopecurus pratensis & $\mathrm{H}$ & sa-ES & $\mathrm{sp}$ & MA & $\mathrm{A}, \mathrm{MA}$ & . & . & $\mathrm{LC}$ & CWR \\
\hline Anagallis arvensis & $\mathrm{T}$ & S-SE Asia & ar & S & $S$ & . & . & . & . \\
\hline Anchusa arvensis & $\mathrm{T}, \mathrm{H}$ & nd & ar & $S$ & S & . & . & . & . \\
\hline Anemone nemorosa & G & sa-ES(d) & $\mathrm{sp}$ & QF & QF & . & an & . & . \\
\hline Anemone ranunculoides & G & $\mathrm{CE}$ & $\mathrm{sp}$ & QF & QF & $9110,9130,9160$ & an & . & . \\
\hline Angelica sylvestris & $\mathrm{H}$ & sa-ES & $\mathrm{sp}$ & MA & MA & . & . & . & . \\
\hline Anthemis arvensis & $\mathrm{T}$ & $\mathrm{N}$-med & ar & S & S & . & . & . & . \\
\hline Anthoxanthum aristatum & $\mathrm{T}$ & Eur $S$ & $\mathrm{kn}$ & S & S & . & . & . & . \\
\hline Anthoxanthum odoratum & $\mathrm{H}$ & sa-ES(d)-M-IR(m) & $\mathrm{sp}$ & - & A, MA, TG & . & . & . & . \\
\hline Anthriscus sylvestris & $\mathrm{H}$ & sa-CE-M & $\mathrm{sp}$ & A & MA, A & . & . & . & . \\
\hline Apera spica-venti & $\mathrm{T}, \mathrm{H}$ & nd & ar & $S$ & S & . & . & . & . \\
\hline Aphanes arvensis & $\mathrm{T}$ & med-atl & ar & S & S & . & . & . & . \\
\hline Arabidopsis thaliana & $\mathrm{T}$ & sa-ES(w)-M(n)-IR(m) & $\mathrm{sp}$ & $\mathrm{S}$ & KC, PP, S & . & . & . & . \\
\hline Arctium lappa & $\mathrm{H}$ & sa-ES(w)-M(n)-IR & $\mathrm{sp}$ & $\mathrm{A}$ & A & . & . & . & . \\
\hline Arctium tomentosum & $\mathrm{H}$ & $\mathrm{ES}(\mathrm{w})-\mathrm{IR}(\mathrm{m})$ & $\mathrm{sp}$ & $\mathrm{A}$ & $\mathrm{A}$ & . & . & . & . \\
\hline Arenaria serpyllifolia & $\mathrm{T}$ & $\mathrm{CB}(\mathrm{d})$ & $\mathrm{sp}$ & FB & $\mathrm{A}, \mathrm{KC}, \mathrm{PP}$ & 6210 & . & . & . \\
\hline Arnoseris minima & $\mathrm{T}$ & CE: sat & $\mathrm{sp}$ & $\mathrm{S}$ & S & . & . & . & . \\
\hline Arrhenatherum elatius & $\mathrm{H}$ & sa-CE-M(n) & $\mathrm{sp}$ & MA & A, MA, TG & 6510 & . & $\mathrm{LC}$ & CWR \\
\hline Artemisia campestris & $\mathrm{Ch}$ & $\mathrm{ES}(\mathrm{w})$ & $\mathrm{sp}$ & $\mathrm{FB}$ & $\mathrm{A}, \mathrm{KC}$ & 6210 & . & . & . \\
\hline Artemisia vulgaris & $\mathrm{H}$ & sa-ES-M(n)-IR(?) & $\mathrm{sp}$ & $\mathrm{A}$ & $\mathrm{A}, \mathrm{MA}, \mathrm{S}$ & . & . & . & . \\
\hline Astragalus glycyphyllos & $\mathrm{H}$ & $E S-(w, d)$ & $\mathrm{sp}$ & TG & TG & . & . & . & . \\
\hline Athyrium filix-femina & $\mathrm{H}$ & $\mathrm{CB}$ & $\mathrm{sp}$ & - & QF & . & an & . & . \\
\hline Atriplex patula & $\mathrm{T}$ & sa-ES(w)-M & $\mathrm{sp}$ & S & S & . & . & . & . \\
\hline Batrachium aquatile & Hy & $\mathrm{CB}(\mathrm{d})$ & $\mathrm{sp}$ & Po & Po & 3260 & . & . & . \\
\hline Bellis perennis & $\mathrm{H}$ & sa-CE-M(n) & $\mathrm{sp}$ & MA & MA & . & . & . & . \\
\hline Berteroa incana & $\mathrm{H}, \mathrm{T}$ & nd & ar & $\mathrm{A}$ & $A, S$ & . & . & . & . \\
\hline Betula pendula & M & sa-ES & $\mathrm{sp}$ & - & KC & . & . & . & . \\
\hline
\end{tabular}


Tab. 1 (continued)

\begin{tabular}{|c|c|c|c|c|c|c|c|c|c|}
\hline 1 & 2 & 3 & 4 & 5 & 6 & 7 & 8 & 9 & 10 \\
\hline Bidens cernua & $\mathrm{T}$ & $\mathrm{CB}(\mathrm{d})$ & $\mathrm{sp}$ & $\mathrm{B}$ & $\mathrm{B}$ & . & . & $\mathrm{LC}$ & . \\
\hline Bidens tripartita & $\mathrm{T}$ & sa-ES-M(n)-IR-Asia SE & $\mathrm{sp}$ & B & $\mathrm{B}, \mathrm{S}$ & . & . & $\mathrm{LC}$ & . \\
\hline Brachypodium sylvaticum & $\mathrm{H}$ & sa-ES(w)-M-IR(m) & $\mathrm{sp}$ & QF & QF & . & an & . & . \\
\hline Bromus carinatus & $\mathrm{T}, \mathrm{H}$ & $\mathrm{Am} \mathrm{N}$ & $\mathrm{kn}$ & - & A & . & . & . & . \\
\hline Bromus hordeaceus & $\mathrm{T}$ & sa-CE-M & $\mathrm{sp}$ & S & A, S & 6510 & . & . & . \\
\hline Bromus inermis & $\mathrm{H}$ & ES & $\mathrm{sp} ?$ & A & A & . & . & . & . \\
\hline Bromus secalinus & $\mathrm{T}, \mathrm{H}$ & ar. antr & ar & $S$ & S & . & . & . & . \\
\hline Calamagrostis canescens & $\mathrm{H}$ & $\mathrm{ES}(\mathrm{w})$ & $\mathrm{sp}$ & $\mathrm{Ph}$ & $\mathrm{Ph}$ & . & . & . & . \\
\hline Calamagrostis epigejos & $\mathrm{G}, \mathrm{H}$ & sa-ES-M(n)-IR-Asia E & $\mathrm{sp}$ & $\mathrm{E}$ & $\mathrm{E}, \mathrm{Q}$ & . & . & . & . \\
\hline Callitriche cophocarpa & $\mathrm{Hy}$ & $\mathrm{ES}(\mathrm{w})$ & $\mathrm{sp}$ & Po & Po & 3260 & . & $\mathrm{LC}$ & . \\
\hline Caltha palustris & $\mathrm{H}$ & $\mathrm{CB}$ & $\mathrm{sp}$ & MA & MA, MC & . & . & $\mathrm{LC}$ & . \\
\hline Campanula patula & $\mathrm{H}$ & $\mathrm{CE}$ & $\mathrm{sp}$ & MA & MA & 6510 & . & . & . \\
\hline Campanula rotundifolia & $\mathrm{H}$ & $\mathrm{CB}(\mathrm{d}): \mathrm{c}-\mathrm{b}-\mathrm{w}$ & $\mathrm{sp}$ & - & Q & . & . & . & . \\
\hline Capsella bursa-pastoris & $\mathrm{H}, \mathrm{T}$ & nd & ar & $\mathrm{PP}$ & MA, S, PP & . & . & . & . \\
\hline Cardamine amara & $\mathrm{H}$ & $\mathrm{CE}$ & $\mathrm{sp}$ & $\mathrm{MC}$ & $\mathrm{MC}, \mathrm{Ph}$ & . & . & $\mathrm{LC}$ & . \\
\hline Cardamine pratensis & $\mathrm{H}$ & $\mathrm{CB}(\mathrm{w}, \mathrm{n})$ & $\mathrm{sp}$ & MA & MA & . & . & $\mathrm{LC}$ & . \\
\hline Cardaminopsis arenosa & $\mathrm{H}$ & $\mathrm{CE}$ & $\mathrm{sp}$ & - & MA & . & . & . & . \\
\hline Carex acutiformis & G, Hy & sa-ES-M(n)-IR(m) & $\mathrm{sp}$ & $\mathrm{Ph}$ & $\mathrm{Ph}$ & . & . & LC & . \\
\hline Carex cespitosa & $\mathrm{H}$ & ES & $\mathrm{sp}$ & MA & MA & . & . & . & . \\
\hline Carex elata & $\mathrm{H}, \mathrm{Hy}$ & sa-CE & $\mathrm{sp}$ & $\mathrm{Ph}$ & $\mathrm{Ph}$ & . & . & $\mathrm{LC}$ & . \\
\hline Carex hirta & G & sa-CE-M(n) & $\mathrm{sp}$ & MA & A, MA & . & . & . & . \\
\hline Carex ovalis & $\mathrm{H}$ & sa-ES(w)-M(w) & $\mathrm{sp}$ & $\mathrm{CU}$ & A & 4030 & . & . & . \\
\hline Carex paniculata & $\mathrm{H}$ & sa-CE & $\mathrm{sp}$ & $\mathrm{Ph}$ & $\mathrm{Ph}$ & . & . & $\mathrm{LC}$ & . \\
\hline Carex pilulifera & $\mathrm{H}$ & sa-CE: ce-b & $\mathrm{sp}$ & Q & Q & 9190 & . & . & . \\
\hline Carex remota & $\mathrm{H}$ & sa-CE-M-IR(m) & $\mathrm{sp}$ & $\mathrm{MC}$ & $\mathrm{MC}$ & . & an & . & . \\
\hline Carex riparia & $\mathrm{H}, \mathrm{Hy}$ & sa-ES-M(n)-IR(n) & $\mathrm{sp}$ & $\mathrm{Ph}$ & $\mathrm{Ph}$ & . & . & $\mathrm{LC}$ & . \\
\hline Carex rostrata & $\mathrm{H}, \mathrm{Hy}$ & $\mathrm{CB}$ & $\mathrm{sp}$ & $\mathrm{Ph}$ & $\mathrm{Ph}$ & 7140 & . & $\mathrm{LC}$ & . \\
\hline Carlina vulgaris & $\mathrm{H}, \mathrm{T}$ & $\mathrm{CE}-\mathrm{M}(\mathrm{n})$ & $\mathrm{sp}$ & $\mathrm{FB}$ & A/MA & 6210 & . & . & . \\
\hline Carpinus betulus & $\mathrm{M}$ & $\mathrm{CE}-\mathrm{M}(\mathrm{n})$ & $\mathrm{sp}$ & QF & QF & 9160 & . & . & . \\
\hline Centaurea cyanus & $\mathrm{T}$ & $\mathrm{N}$-med & ar & $S$ & S & . & . & . & . \\
\hline Centaurea scabiosa & $\mathrm{H}$ & sa-ES(w) & $\mathrm{sp}$ & $\mathrm{FB}$ & A & 6210 & . & . & . \\
\hline Centaurium erythraea & $\mathrm{T}, \mathrm{H}$ & sa-CE-M-IR & $\mathrm{sp}$ & - & MA & . & . & . & . \\
\hline Cerastium arvense & $\mathrm{C}$ & $\mathrm{CB}$ & $\mathrm{sp}$ & $\mathrm{KC}$ & MA & . & . & . & . \\
\hline Cerastium holosteoides & $\mathrm{C}, \mathrm{H}$ & sa-ES-M-IR & $\mathrm{sp}$ & MA & MA & . & . & . & . \\
\hline Chaerophyllum temulum & $\mathrm{T}, \mathrm{H}$ & sa-CE-M & $\mathrm{sp}$ & A & $\mathrm{A}, \mathrm{QF}$ & . & . & . & . \\
\hline Chamaenerion angustifolium & $\mathrm{H}$ & $\mathrm{CB}$ & $\mathrm{sp}$ & $\mathrm{E}$ & Q & . & . & . & . \\
\hline Chamomilla suaveolens & $\mathrm{T}$ & Am N \& Asia E & $\mathrm{kn}$ & PP & A, PP, S & . & . & . & . \\
\hline Chelidonium majus & $\mathrm{H}$ & sa-ES-M & $\mathrm{sp}$ & $\mathrm{A}$ & $\mathrm{A}, \mathrm{QF}$ & . & . & . & . \\
\hline Chenopodium album & $\mathrm{T}$ & ES & $\mathrm{sp}$ & S & A, S & . & . & . & . \\
\hline Chenopodium strictum & $\mathrm{T}$ & Asia C & $\mathrm{kn}$ & S & S & . & . & . & . \\
\hline Chrysosplenium alternifolium & $\mathrm{H}$ & ES & $\mathrm{sp}$ & $\mathrm{MC}$ & $\mathrm{MC}$ & . & an & . & . \\
\hline Cirsium arvense & G & sa-ES-M(w)-IR & $\mathrm{sp}$ & A & $\mathrm{A}, \mathrm{MA}, \mathrm{S}$ & . & . & . & . \\
\hline Cirsium oleraceum & $\mathrm{H}$ & $\mathrm{ES}(\mathrm{w})$ & $\mathrm{sp}$ & MA & A, MA & . & . & . & . \\
\hline Cirsium palustre & $\mathrm{H}$ & sa-ES(w) & $\mathrm{sp}$ & MA & MA & . & . & . & . \\
\hline Cirsium vulgare & $\mathrm{H}$ & nd & ar & $\mathrm{A}$ & A & . & . & . & . \\
\hline
\end{tabular}


Tab. 1 (continued)

\begin{tabular}{|c|c|c|c|c|c|c|c|c|c|}
\hline 1 & 2 & 3 & 4 & 5 & 6 & 7 & 8 & 9 & 10 \\
\hline Comarum palustre & $\mathrm{C}$ & $\mathrm{CB}$ & $\mathrm{sp}$ & SC & $\mathrm{Ph}$ & 7140 & . & . & . \\
\hline Convallaria majalis & G & sa-ES(d, w) & $\mathrm{sp}$ & - & QF & . & an & . & . \\
\hline Convolvulus arvensis & $\mathrm{G}, \mathrm{H}, \mathrm{li}$ & sa-ES-M-IR & $\mathrm{sp}$ & A & A, S & . & . & . & . \\
\hline Conyza canadensis & $\mathrm{T}, \mathrm{H}$ & $\mathrm{Am} \mathrm{N}$ & $\mathrm{kn}$ & S & A, S & . & . & . & . \\
\hline Corylus avellana & $\mathrm{N}$ & sa-CE-M(n)-IR(w) & $\mathrm{sp}$ & $\mathrm{RP}$ & QF, RP & . & an & . & . \\
\hline Corynephorus canescens & $\mathrm{H}$ & $\mathrm{CE}(\mathrm{w})$ & $\mathrm{sp}$ & $\mathrm{KC}$ & $\mathrm{KC}$ & . & . & . & . \\
\hline Crataegus monogyna & $\mathrm{N}$ & sa-CE-M-IR(w) & $\mathrm{sp}$ & $\mathrm{RP}$ & QF, RP & . & . & . & . \\
\hline Crataegus rhipidophylla & $\mathrm{N}$ & CE-M(e)-IR(w) & $\mathrm{sp}$ & $\mathrm{RP}$ & $\mathrm{RP}$ & . & . & . & . \\
\hline Crepis capillaris & $\mathrm{H}, \mathrm{T}$ & $\mathrm{CE}(\mathrm{w})-\mathrm{M}(\mathrm{n}, \mathrm{w})$ & $\mathrm{sp}$ & MA & $\mathrm{A}, \mathrm{MA}$ & 6510 & . & . & . \\
\hline Crepis paludosa & $\mathrm{H}$ & $\mathrm{CE}$ & $\mathrm{sp}$ & MA & $\mathrm{MA}, \mathrm{MC}$ & . & . & . & . \\
\hline Crepis tectorum & $\mathrm{H}, \mathrm{T}$ & ES & $\mathrm{sp}$ & $S$ & $S$ & . & . & . & . \\
\hline Dactylis glomerata & $\mathrm{H}$ & sa-ES(d)-M-IR & $\mathrm{sp}$ & MA & $\mathrm{A}, \mathrm{E}, \mathrm{MA}$ & 6510 & . & . & . \\
\hline Daucus carota & $\mathrm{H}$ & sa-CE-M-IR & $\mathrm{sp}$ & A & A, MA & . & . & LC & CWR \\
\hline Deschampsia caespitosa & $\mathrm{H}$ & ES-IR-Asia SE & $\mathrm{sp}$ & MA & MA & . & . & . & . \\
\hline Deschampsia flexuosa & $\mathrm{H}$ & CB: c-b-o & $\mathrm{sp}$ & - & Q & . & . & . & . \\
\hline Digitaria ischaemum & $\mathrm{T}$ & nd & ar & $S$ & $S$ & . & . & . & . \\
\hline Dryopteris carthusiana & $\mathrm{H}$ & $\mathrm{CB}(\mathrm{d})$ & $\mathrm{sp}$ & - & $\mathrm{Q}, \mathrm{QF}$ & . & an & . & . \\
\hline Dryopteris dilatata & $\mathrm{H}$ & sa-(CE) & $\mathrm{sp}$ & VP & Q & . & an & . & . \\
\hline Dryopteris filix-mas & $\mathrm{H}$ & $\mathrm{CB}(\mathrm{d})$ & $\mathrm{sp}$ & QF & $\mathrm{Q}, \mathrm{QF}$ & . & an & . & . \\
\hline Echinochloa crus-galli & $\mathrm{T}$ & S-SE Asia & ar & $S$ & $S$ & . & . & . & . \\
\hline Eleocharis uniglumis & Hy, G & CB: c-b-w & $\mathrm{sp}$ & $\mathrm{J}$ & MA & . & . & $\mathrm{LC}$ & . \\
\hline Elymus repens & G & sa-ES-M-IR & $\mathrm{sp}$ & A & A, MA, RP, S & . & . & . & . \\
\hline Epilobium hirsutum & $\mathrm{H}$ & sa-ES(w)-M-IR & $\mathrm{sp}$ & A & A, MA & 6430 & . & . & . \\
\hline Epilobium parviflorum & $\mathrm{H}$ & sa-CE-M-IR(w) & $\mathrm{sp}$ & A & A, MA & 6430 & . & . & . \\
\hline Equisetum arvense & G & $\mathrm{CB}$ & $\mathrm{sp}$ & A & $\mathrm{A}, \mathrm{S}$ & . & . & LC & . \\
\hline Equisetum fluviatile & Hy, G & $\mathrm{CB}$ & $\mathrm{sp}$ & $\mathrm{Ph}$ & MA & . & . & $\mathrm{LC}$ & . \\
\hline Equisetum hyemale & $\mathrm{C}$ & $\mathrm{CB}$ & $\mathrm{sp}$ & - & QF & . & an & . & . \\
\hline Equisetum palustre & G & $\mathrm{CB}$ & $\mathrm{sp}$ & MA & A, MA & . & . & $\mathrm{LC}$ & . \\
\hline Equisetum sylvaticum & G & $\mathrm{CB}$ & $\mathrm{sp}$ & QF & QF & $* 91 \mathrm{E} 0,91 \mathrm{~F} 0$ & an & . & . \\
\hline Erigeron annuus & $\mathrm{H}, \mathrm{T}$ & $\mathrm{Am} \mathrm{N}$ & $\mathrm{kn}$ & A & A, S & . & . & . & . \\
\hline Erodium cicutarium & $\mathrm{T}, \mathrm{H}$ & sa-ES-M-IR & $\mathrm{sp}$ & S & $S$ & . & . & . & . \\
\hline Erophila verna & $\mathrm{T}$ & sa-ES(w)-M-IR & $\mathrm{sp}$ & S & A, KC, S & . & . & . & . \\
\hline Erysimum cheiranthoides & $\mathrm{T}$ & sa-ES & $\mathrm{sp}$ & S & $A, S$ & . & . & . & . \\
\hline Euonymus europaea & $\mathrm{N}$ & sa-CE-M(n) & $\mathrm{sp}$ & $\mathrm{RP}$ & QF, RP & . & an & . & . \\
\hline Euphorbia helioscopia & $\mathrm{T}$ & med & ar & S & $S$ & . & . & . & . \\
\hline Euphrasia stricta & $\mathrm{T}, \mathrm{pp}$ & $\mathrm{ES}(\mathrm{w})$ & $\mathrm{sp}$ & FB & $\mathrm{A} / \mathrm{MA}$ & 6210 & . & . & . \\
\hline Fagus sylvatica & $\mathrm{M}$ & sa-CE $(w): c e-b$ & $\mathrm{sp}$ & QF & Q & 9110,9130 & . & . & . \\
\hline Fallopia convolvulus & $\mathrm{T}, \mathrm{H}$ & nd & ar & S & S & 9110,9130 & . & . & . \\
\hline Fallopia dumetorum & $\mathrm{T}$ & sa-ES-M(n) & $\mathrm{sp}$ & A & A & 6430 & . & . & . \\
\hline Festuca gigantea & $\mathrm{H}$ & sa-ES(w)-IR(m) & $\mathrm{sp}$ & QF & QF & ${ }^{*} 91 \mathrm{E} 0,91 \mathrm{~F} 0$ & an & . & . \\
\hline Festuca pratensis & $\mathrm{H}$ & sa-ES-IR(m) & $\mathrm{sp}$ & MA & MA & . & . & . & . \\
\hline Festuca rubra & $\mathrm{H}$ & $\mathrm{CB}$ & $\mathrm{sp}$ & MA & $\mathrm{A}, \mathrm{MA}, \mathrm{Q}$ & . & . & $\mathrm{LC}$ & CWR \\
\hline Festuca trachyphylla & $\mathrm{H}$ & sa-CE(w) & $\mathrm{sp}$ & $\mathrm{FB}$ & A/MA & 6210 & . & . & . \\
\hline Ficaria verna & G & sa-CE-M? & $\mathrm{sp}$ & QF & QF & $9110,9130,9160$ & an & . & . \\
\hline Filago minima & $\mathrm{T}$ & sa-CE: ce-b & $\mathrm{sp}$ & KC & $\mathrm{KC}, \mathrm{S}$ & . & . & . & . \\
\hline
\end{tabular}


Tab. 1 (continued)

\begin{tabular}{|c|c|c|c|c|c|c|c|c|c|}
\hline 1 & 2 & 3 & 4 & 5 & 6 & 7 & 8 & 9 & 10 \\
\hline Filipendula ulmaria & $\mathrm{H}$ & sa-ES & $\mathrm{sp}$ & MA & A, MA & . & . & . & . \\
\hline Fragaria vesca & $\mathrm{H}$ & $\mathrm{CB}$ & $\mathrm{sp}$ & TG & TG & . & . & $\mathrm{LC}$ & CWR \\
\hline Frangula alnus & $\mathrm{N}$ & sa-ES(w) & $\mathrm{sp}$ & $\mathrm{RP}$ & $\mathrm{RP}$ & . & . & . & . \\
\hline Fraxinus excelsior & M & sa-CE-M(n)-IR(w) & $\mathrm{sp}$ & QF & QF & $9110,9130,9160$ & . & . & . \\
\hline Fumaria officinalis & $\mathrm{T}$ & med & ar & $\mathrm{S}$ & S & . & . & . & . \\
\hline Gagea lutea & G & $\mathrm{ES}(\mathrm{d})$ & $\mathrm{sp}$ & QF & QF & 91F0 & an & . & . \\
\hline Galeobdolon luteum & $\mathrm{C}$ & CE: ece & $\mathrm{sp}$ & QF & $\mathrm{QF}$ & $9110,9130,9160$ & an & . & . \\
\hline Galeopsis pubescens & $\mathrm{T}$ & CE & $\mathrm{sp}$ & A & $\mathrm{A}, \mathrm{QF}$ & . & . & . & . \\
\hline Galeopsis tetrahit & $\mathrm{T}$ & sa-CE & $\mathrm{sp}$ & A & $\mathrm{A}, \mathrm{S}$ & . & . & . & . \\
\hline Galinsoga ciliata & $\mathrm{T}$ & $\mathrm{Am} \mathrm{C}$ & $\mathrm{kn}$ & S & S & . & . & . & . \\
\hline Galinsoga parviflora & $\mathrm{T}$ & Am S \& C & $\mathrm{kn}$ & S & $\mathrm{S}$ & . & . & . & . \\
\hline Galium aparine & $\mathrm{T}, \mathrm{H}$ & $\mathrm{CB}(\mathrm{d})$ & $\mathrm{sp}$ & A & $\mathrm{A}, \mathrm{S}$ & . & . & . & . \\
\hline Galium mollugo & $\mathrm{H}$ & sa-CE-M(n) & $\mathrm{sp}$ & MA & MA & 6510 & . & . & . \\
\hline Galium palustre & $\mathrm{H}$ & $\mathrm{CB}(\mathrm{d})$ & $\mathrm{sp}$ & $\mathrm{Ph}$ & $\mathrm{Ph}$ & . & . & . & . \\
\hline Galium verum & $\mathrm{H}$ & sa-ES-M(n)-IR & $\mathrm{sp}$ & $\mathrm{FB}$ & A, MA & 6210 & . & . & . \\
\hline Geranium pratense & $\mathrm{H}$ & ES & $\mathrm{sp}$ & MA & MA & 6510 & . & . & . \\
\hline Geranium pusillum & $\mathrm{T}$ & iranoanatol & ar & S & $\mathrm{A}, \mathrm{S}$ & . & . & . & . \\
\hline Geranium robertianum & $\mathrm{H}, \mathrm{T}$ & sa-CE-M-IR & $\mathrm{sp}$ & A & $\mathrm{A}, \mathrm{QF}$ & . & . & . & . \\
\hline Geum rivale & $\mathrm{H}$ & $\mathrm{CB}(\mathrm{d})$ & $\mathrm{sp}$ & MA & MA & . & an & . & . \\
\hline Geum urbanum & $\mathrm{H}$ & sa-ES(w)-M-IR(m) & $\mathrm{sp}$ & A & $\mathrm{A}, \mathrm{QF}$ & . & an & . & . \\
\hline Glechoma hederacea & $\mathrm{G}, \mathrm{H}$ & sa-ES-M(n, w) & $\mathrm{sp}$ & A & A, MA & . & . & . & . \\
\hline Glyceria fluitans & Hy & sa-CE-M(n) & $\mathrm{sp}$ & $\mathrm{Ph}$ & $\mathrm{Ph}$ & . & . & $\mathrm{LC}$ & . \\
\hline Glyceria notata & Hy & sa-CE-M-IR(m) & $\mathrm{sp}$ & $\mathrm{Ph}$ & $\mathrm{Ph}$ & . & . & $\mathrm{LC}$ & . \\
\hline Gnaphalium sylvaticum & $\mathrm{H}$ & sa-ES(w) & $\mathrm{sp}$ & $\mathrm{E}$ & $\mathrm{A}, \mathrm{E}$ & . & . & . & . \\
\hline Gnaphalium uliginosum & $\mathrm{T}$ & sa-ES & $\mathrm{sp}$ & IJ & $\mathrm{B}, \mathrm{S}$ & . & . & . & . \\
\hline Hedera helix & $\mathrm{N}, \mathrm{Ch}$ & sa-CE(w)-M & $\mathrm{sp}$ & QF & QF & . & an & . & . \\
\hline Helianthus tuberosus & G & $A m N$ & $\mathrm{kn}$ & A & $\mathrm{A}$ & . & . & . & . \\
\hline Helichrysum arenarium & $\mathrm{H}$ & $\mathrm{ES}(\mathrm{w})-\mathrm{IR}(\mathrm{m})$ & $\mathrm{sp}$ & $\mathrm{KC}$ & $\mathrm{KC}$ & . & . & . & . \\
\hline Heracleum sibiricum & $\mathrm{H}$ & $\mathrm{ES}(\mathrm{w})$ & $\mathrm{sp}$ & A & A, MA & . & . & . & . \\
\hline Herniaria glabra & $\mathrm{H}$ & sa-ES(w)-M-IR(n) & $\mathrm{sp}$ & $\mathrm{PP}$ & $\mathrm{PP}$ & . & . & . & . \\
\hline Hieracium pilosella & $\mathrm{H}$ & sa-CE-M & $\mathrm{sp}$ & $\mathrm{CU}$ & A/MA, S & 4030 & . & . & . \\
\hline Hieracium umbellatum & $\mathrm{H}$ & $\mathrm{CB}$ & $\mathrm{sp}$ & Q & Q & 9190 & . & . & . \\
\hline Holcus lanatus & $\mathrm{H}$ & sa-CE-M & $\mathrm{sp}$ & MA & A, MA & . & . & . & . \\
\hline Holcus mollis & $\mathrm{G}, \mathrm{H}$ & sa-CE: ce-b & $\mathrm{sp}$ & Q & Q, S & 9190 & . & . & . \\
\hline Humulus lupulus & $\mathrm{H}$, li & $\mathrm{CB}(\mathrm{d})$ & $\mathrm{sp}$ & A & $\mathrm{A}, \mathrm{RP}$ & 6430 & . & . & . \\
\hline Hypericum perforatum & $\mathrm{H}$ & sa-ES(w)-M-IR & $\mathrm{sp}$ & TG & MA, TG & . & . & . & . \\
\hline Hypericum tetrapterum & $\mathrm{H}$ & sa-CE-M & $\mathrm{sp}$ & MA & MA & . & . & . & . \\
\hline Hypochoeris radicata & $\mathrm{H}$ & sa-CE-M & $\mathrm{sp}$ & $\mathrm{KC}$ & $\mathrm{A} / \mathrm{MA}, \mathrm{KC}$ & . & . & . & . \\
\hline Impatiens noli-tangere & $\mathrm{T}$ & $\mathrm{CB}(\mathrm{d})$ & $\mathrm{sp}$ & A & $\mathrm{A}, \mathrm{QF}$ & . & an & . & . \\
\hline Iris pseudacorus & G, Hy & sa-CE-M-IR(w) & $\mathrm{sp}$ & $\mathrm{Ph}$ & $\mathrm{Ph}$ & . & . & $\mathrm{LC}$ & . \\
\hline Jasione montana & $\mathrm{H}$ & sa-CE-M(w) & $\mathrm{sp}$ & $\mathrm{KC}$ & A/MA & . & . & . & . \\
\hline Juncus articulatus & $\mathrm{H}$ & $\mathrm{CB}$ & $\mathrm{sp}$ & SC & MA & 7140 & . & $\mathrm{LC}$ & . \\
\hline Juncus bufonius & $\mathrm{T}$ & $\mathrm{CB}$ & $\mathrm{sp}$ & IJ & $\mathrm{B}, \mathrm{S}$ & . & . & $\mathrm{LC}$ & . \\
\hline Juncus effusus & $\mathrm{H}$ & cosmopolitan & $\mathrm{sp}$ & MA & MA & . & . & $\mathrm{LC}$ & . \\
\hline Juniperus communis & $\mathrm{N}$ & CB: $c-b-w$ & $\mathrm{sp}$ & VP & Q & . & . & . & . \\
\hline
\end{tabular}


Tab. 1 (continued)

\begin{tabular}{|c|c|c|c|c|c|c|c|c|c|}
\hline 1 & 2 & 3 & 4 & 5 & 6 & 7 & 8 & 9 & 10 \\
\hline Knautia arvensis & $\mathrm{H}$ & sa-ES(w)-M & $\mathrm{sp}$ & TG & MA, TG & . & . & . & . \\
\hline Lamium amplexicaule & $\mathrm{T}$ & med-iranotur & ar & $\mathrm{S}$ & $\mathrm{S}$ & . & . & . & . \\
\hline Lamium maculatum & $\mathrm{H}$ & CE-M(n) & $\mathrm{sp}$ & A & A & . & . & . & . \\
\hline Lapsana communis & $\mathrm{H}, \mathrm{T}$ & sa-CE-M(n) & $\mathrm{sp}$ & $\mathrm{A}$ & A, QF, S & . & . & . & . \\
\hline Lathyrus montanus & G & sa-CE-M(n) & $\mathrm{sp}$ & Q & Q & 9190 & an & . & . \\
\hline Lathyrus pratensis & $\mathrm{H}$ & sa-ES-M-IR & $\mathrm{sp}$ & MA & MA & . & . & . & . \\
\hline Lathyrus sylvestris & $\mathrm{H}$ & sa-CE-M(n) & $\mathrm{sp}$ & TG & TG & . & . & $\mathrm{LC}$ & CWR \\
\hline Lemna minor & Hy & cosmopolitan & $\mathrm{sp}$ & $\mathrm{L}$ & $\mathrm{L}$ & . & . & $\mathrm{LC}$ & . \\
\hline Leontodon autumnalis & $\mathrm{H}$ & sa-ES(w) & $\mathrm{sp}$ & MA & MA & . & . & . & . \\
\hline Leontodon hispidus & $\mathrm{H}$ & CE-M(n)-IR: ia & $\mathrm{sp}$ & MA & MA & 6510 & . & . & . \\
\hline Leonurus cardiaca & $\mathrm{H}$ & pont & ar & A & $\mathrm{A}$ & . & . & . & . \\
\hline Linaria vulgaris & G & sa-ES & $\mathrm{sp}$ & A & MA & . & . & . & . \\
\hline Lithospermum arvense & $\mathrm{T}$ & med-iranotur & ar & S & S & . & . & . & . \\
\hline Lolium perenne & $\mathrm{H}$ & sa-CE-M-IR(w) & $\mathrm{sp}$ & MA & A, MA & . & . & $\mathrm{LC}$ & CWR \\
\hline Lotus uliginosus & $\mathrm{H}$ & sa-CE(w)-M(n) & $\mathrm{sp}$ & MA & MA & . & . & . & . \\
\hline Luzula campestris & $\mathrm{H}$ & sa-CE-M(n) & $\mathrm{sp}$ & $\mathrm{CU}$ & MA & 4030 & . & . & . \\
\hline Lycopus europaeus & $\mathrm{H}, \mathrm{Hy}$ & sa-ES(w)-M(n)-IR & $\mathrm{sp}$ & $\mathrm{Ag}$ & $\mathrm{A}, \mathrm{Ag}, \mathrm{B}, \mathrm{Ph}$ & . & . & $\mathrm{LC}$ & . \\
\hline Lysimachia vulgaris & $\mathrm{H}$ & sa-ES-M(n)-IR(m) & $\mathrm{sp}$ & MA & A, MA & . & . & $\mathrm{LC}$ & . \\
\hline Lythrum salicaria & $\mathrm{H}$ & sa-ES(d)-M-IR & $\mathrm{sp}$ & MA & $\mathrm{A}, \mathrm{MA}, \mathrm{Ph}$ & . & . & $\mathrm{LC}$ & . \\
\hline Maianthemum bifolium & G & ES & $\mathrm{sp}$ & - & $\mathrm{Q}, \mathrm{QF}$ & . & an & . & . \\
\hline Malva neglecta & $\mathrm{H}, \mathrm{T}$ & iranoanatol & ar & S & S & . & . & . & . \\
\hline $\begin{array}{l}\text { Matricaria maritima subsp. } \\
\text { inodora }\end{array}$ & $\mathrm{H}, \mathrm{T}$ & ar. resist & ar & S & A, S & . & . & . & . \\
\hline Medicago lupulina & $\mathrm{H}, \mathrm{T}$ & sa-ES-M-IR & $\mathrm{sp}$ & A & A, MA & . & . & $\mathrm{LC}$ & CWR \\
\hline Melampyrum pratense & $\mathrm{T}, \mathrm{pp}$ & sa-ES(w) & $\mathrm{sp}$ & Q & $\mathrm{Q}, \mathrm{TG}$ & 9190 & an & . & . \\
\hline Melandrium album & $\mathrm{T}, \mathrm{H}$ & nd & ar & A & A & . & . & . & . \\
\hline Mentha aquatica & $\mathrm{H}, \mathrm{Hy}$ & sa-ES(w)-M & $\mathrm{sp}$ & $\mathrm{Ph}$ & $\mathrm{Ph}$ & . & . & $\mathrm{LC}$ & . \\
\hline Mentha xverticillata & $\mathrm{H}$ & sa-ES(w)-M [?] & $\mathrm{sp}$ & - & $\mathrm{MA}, \mathrm{S}, \mathrm{PP}$ & . & . & . & . \\
\hline Milium effusum & $\mathrm{H}$ & $\mathrm{CB}(\mathrm{d})$ & $\mathrm{sp}$ & QF & QF & . & an & . & . \\
\hline Mycelis muralis & $\mathrm{H}$ & $\mathrm{CE}$ & $\mathrm{sp}$ & A & QF & . & an & . & . \\
\hline Myosotis arvensis & $\mathrm{T}, \mathrm{H}$ & med-C Asia & ar & $\mathrm{S}$ & S & . & . & . & . \\
\hline Myosotis palustris & $\mathrm{H}$ & sa-ES-M(n) & $\mathrm{sp}$ & MA & $\mathrm{A}, \mathrm{MA}, \mathrm{Ph}$ & . & . & . & . \\
\hline Myosurus minimus & $\mathrm{T}$ & $\mathrm{CB}(\mathrm{d})$ & $\mathrm{sp}$ & IJ & IJ, S & . & . & . & . \\
\hline Oenanthe aquatica & $\mathrm{H}, \mathrm{Hy}$ & sa-ES(w) & $\mathrm{sp}$ & $\mathrm{Ph}$ & $\mathrm{Ph}$ & . & . & . & . \\
\hline Oxalis acetosella & $\mathrm{G}, \mathrm{H}$ & sa-ES & $\mathrm{sp}$ & - & QF & . & an & . & . \\
\hline Oxalis fontana & G & Am N \& Asia E? & $\mathrm{kn}$ & S & A, S & . & . & . & . \\
\hline Padus avium & $\mathrm{M}$ & ES & $\mathrm{sp}$ & QF & QF & ${ }^{*} 91 \mathrm{E} 0,91 \mathrm{~F} 0$ & . & $\mathrm{LC}$ & CWR \\
\hline Papaver argemone & $\mathrm{T}$ & med-W-iranoanatol & ar & $\mathrm{S}$ & S & . & . & . & . \\
\hline Papaver dubium & $\mathrm{T}$ & med-iranoanatol & ar & $S$ & $S$ & . & . & . & . \\
\hline Peucedanum oreoselinum & $\mathrm{H}$ & CE-M(n) & $\mathrm{sp}$ & TG & TG & . & . & . & . \\
\hline Phalaris arundinacea & $\mathrm{G}, \mathrm{H}$ & $\mathrm{CB}$ & $\mathrm{sp}$ & $\mathrm{Ph}$ & $\mathrm{A}, \mathrm{Ph}$ & *91E0 & . & $\mathrm{LC}$ & . \\
\hline Phleum pratense & $\mathrm{H}$ & sa-ES-M & $\mathrm{sp}$ & MA & MA & . & . & $\mathrm{LC}$ & CWR \\
\hline Picea abies & $\mathrm{M}$ & CE: a-ne & $\mathrm{sp}$ & VP & Q & . & . & . & . \\
\hline Picris hieracioides & $\mathrm{H}$ & sa-ES-Asia E & $\mathrm{sp}$ & $\mathrm{A}$ & A & . & . & . & . \\
\hline Pimpinella saxifraga & $\mathrm{H}$ & sa-ES(w)-M(n) & $\mathrm{sp}$ & $\mathrm{FB}$ & $\mathrm{A} / \mathrm{MA}$ & 6210 & . & . & . \\
\hline Pinus sylvestris & $\mathrm{M}$ & ES & $\mathrm{sp}$ & VP & Q & . & . & . & . \\
\hline
\end{tabular}


Tab. 1 (continued)

\begin{tabular}{|c|c|c|c|c|c|c|c|c|c|}
\hline 1 & 2 & 3 & 4 & 5 & 6 & 7 & 8 & 9 & 10 \\
\hline Plantago lanceolata & $\mathrm{H}$ & sa-ES(w)-M-IR & $\mathrm{sp}$ & MA & A, MA, S & . & . & . & . \\
\hline Plantago major & $\mathrm{H}$ & cosmopolitan & $\mathrm{sp}$ & MA & A, MA, PP, S & . & . & . & . \\
\hline Platanthera bifolia & G & sa-ES-M & $\mathrm{sp}$ & QF & QF & . & . & $\mathrm{LC}$ & . \\
\hline Poa angustifolia & $\mathrm{H}$ & $\mathrm{CB}$ & $\mathrm{sp}$ & TG & A & . & . & . & . \\
\hline Poa апnиа & $\mathrm{H}, \mathrm{T}$ & sa-ES-M-IR-Asia E & $\mathrm{sp}$ & $\mathrm{PP}$ & PP, S & . & . & . & . \\
\hline Poa nemoralis & $\mathrm{H}$ & sa-ES-M-IR & $\mathrm{sp}$ & QF & QF & . & an & . & . \\
\hline Poa palustris & $\mathrm{H}$ & $\mathrm{CB}$ & $\mathrm{sp}$ & MA & MA & . & . & . & . \\
\hline Poa pratensis & $\mathrm{H}$ & CB & $\mathrm{sp}$ & MA & MA & . & . & $\mathrm{LC}$ & CWR \\
\hline Poa trivialis & $\mathrm{H}$ & sa-ES-M(?)-IR(?) & $\mathrm{sp}$ & MA & $\mathrm{A}, \mathrm{MA}, \mathrm{QF}$ & . & . & . & . \\
\hline Polygonatum multiflorum & G & sa-CE-M(n)-IR(m) & $\mathrm{sp}$ & QF & QF & $9110,9130,9160$ & an & . & . \\
\hline Polygonum amphibium & Hy, G & $\mathrm{CB}$ & $\mathrm{sp}$ & - & $\mathrm{A}, \mathrm{B}, \mathrm{MA}, \mathrm{Ph}$ & . & . & . & . \\
\hline Polygonum aviculare & $\mathrm{T}$ & cosmopolitan & $\mathrm{sp}$ & - & A, B, MA, S & . & . & . & . \\
\hline Polygonum hydropiper & $\mathrm{T}$ & sa-ES-M-IR-Asia SE & $\mathrm{sp}$ & $\mathrm{B}$ & $\mathrm{S}$ & . & . & . & . \\
\hline $\begin{array}{l}\text { Polygonum lapathifolium } \\
\text { subsp. lapathifolium }\end{array}$ & $\mathrm{T}$ & cosmopolitan & $\mathrm{sp}$ & $\mathrm{B}$ & B & . & . & . & . \\
\hline $\begin{array}{l}\text { Polygonum lapathifolium } \\
\text { subsp. pallidum }\end{array}$ & $\mathrm{T}$ & cosmopolitan & ar & S & S & . & . & . & . \\
\hline Polygonum minus & $\mathrm{T}$ & sa-ES & $\mathrm{sp}$ & B & $S$ & . & . & . & . \\
\hline Polygonum persicaria & $\mathrm{T}$ & sa-ES(d)-M-IR & $\mathrm{sp}$ & $\mathrm{S}$ & S & . & . & . & . \\
\hline Populus nigra & $\mathrm{M}$ & $\mathrm{ES}(\mathrm{w})-\mathrm{M}-\mathrm{IR}(\mathrm{w})$ & $\mathrm{sp}$ & Sp & $\mathrm{RP}$ & ${ }^{*} 91 \mathrm{E} 0$ & . & . & . \\
\hline Populus tremula & $\mathrm{M}$ & sa-ES-M-IR-Asia E & $\mathrm{sp}$ & $\mathrm{RP}$ & $\mathrm{RP}$ & . & . & . & . \\
\hline Potamogeton natans & Hy & CB: $c-b-w$ & $\mathrm{sp}$ & Po & Po & 3150 & . & $\mathrm{LC}$ & . \\
\hline Potentilla anserina & $\mathrm{H}$ & $\mathrm{CB}$ & $\mathrm{sp}$ & MA & $\mathrm{A}, \mathrm{B}, \mathrm{MA}, \mathrm{S}$ & . & . & . & . \\
\hline Potentilla argentea & $\mathrm{H}$ & sa-ES(w)-M(n) & $\mathrm{sp}$ & $\mathrm{KC}$ & $\mathrm{KC}$ & . & . & . & . \\
\hline Potentilla heptaphylla & $\mathrm{H}$ & CE: ce-b & $\mathrm{sp}$ & $\mathrm{FB}$ & $\mathrm{A} / \mathrm{MA}$ & 6210 & . & . & . \\
\hline Potentilla reptans & $\mathrm{H}$ & ES(w)-M-IR & $\mathrm{sp}$ & MA & MA & . & . & . & . \\
\hline Prunella vulgaris & $\mathrm{H}$ & sa-ES(w)-M-IR & $\mathrm{sp}$ & MA & MA & . & . & . & . \\
\hline Pteridium aquilinum & G & cosmopolitan & $\mathrm{sp}$ & Q & Q & 9190 & an & . & . \\
\hline Pyrus pyraster & $\mathrm{M}$ & sa-CE-M(n)-IR: ia & $\mathrm{sp}$ & $\mathrm{RP}$ & MA, RP & . & . & . & . \\
\hline Quercus petraea & $\mathrm{M}$ & sa-CE-M(n) & $\mathrm{sp}$ & - & Q & . & . & . & . \\
\hline Quercus robur & $\mathrm{M}$ & sa-CE-M(n) & $\mathrm{sp}$ & - & $\mathrm{Q}, \mathrm{QF}, \mathrm{RP}$ & . & . & . & . \\
\hline Ranunculus acris & $\mathrm{H}$ & sa-ES(w)-M(n) & $\mathrm{sp}$ & MA & MA & . & . & . & . \\
\hline Ranunculus auricomus & $\mathrm{H}$ & $\mathrm{CE}$ & $\mathrm{sp}$ & QF & QF & 9160 & an & . & . \\
\hline Ranunculus flammula & $\mathrm{H}$ & sa-ES(w)-M(n) & $\mathrm{sp}$ & SC & MA & 7140 & . & LC & . \\
\hline Ranunculus repens & $\mathrm{H}$ & sa-ES-M & $\mathrm{sp}$ & MA & MA & . & . & $\mathrm{LC}$ & . \\
\hline Raphanus raphanistrum & $\mathrm{T}$ & med & ar & $\mathrm{S}$ & $\mathrm{S}$ & . & . & . & . \\
\hline Rhamnus cathartica & $\mathrm{N}$ & $\mathrm{ES}(\mathrm{w})-\mathrm{M}(\mathrm{n})-\mathrm{IR}(\mathrm{m})$ & $\mathrm{sp}$ & $\mathrm{RP}$ & QF & . & an & . & . \\
\hline Ribes alpinum & $\mathrm{N}$ & CE: a-ne & $\mathrm{sp}$ & QF & QF & ${ }^{*} 9180$ & . & . & . \\
\hline Ribes nigrum & $\mathrm{N}$ & ES & $\mathrm{sp}$ & $\mathrm{Ag}$ & $\mathrm{Ag}$ & . & an & . & . \\
\hline Rorippa palustris & $\mathrm{T}, \mathrm{H}$ & $\mathrm{CB}$ & $\mathrm{sp}$ & $\mathrm{B}$ & $\mathrm{B}, \mathrm{S}$ & . & . & $\mathrm{LC}$ & CWR \\
\hline Rosa canina & $\mathrm{N}, \mathrm{li}$ & sa-CE-M-IR & $\mathrm{sp}$ & $\mathrm{RP}$ & $\mathrm{RP}$ & . & . & . & . \\
\hline Rubus idaeus & $\mathrm{N}$ & $\mathrm{CB}$ & $\mathrm{sp}$ & $\mathrm{E}$ & $\mathrm{E}$ & . & . & . & . \\
\hline Rumex acetosa & $\mathrm{H}$ & ES & $\mathrm{sp}$ & MA & A, MA, S & . & . & . & . \\
\hline Rumex acetosella & $\mathrm{G}, \mathrm{H}, \mathrm{T}$ & sa-ES-M & $\mathrm{sp}$ & $\mathrm{KC}$ & $\mathrm{KC}, \mathrm{S}$ & . & . & . & . \\
\hline Rumex crispus & $\mathrm{H}$ & ES-M-IR(m) & $\mathrm{sp}$ & MA & MA & . & . & . & . \\
\hline Rumex obtusifolius & $\mathrm{H}$ & sa-CE-M(n)-IR(w) & $\mathrm{sp}$ & A & A, MA & . & . & . & . \\
\hline
\end{tabular}


Tab. 1 (continued)

\begin{tabular}{|c|c|c|c|c|c|c|c|c|c|}
\hline 1 & 2 & 3 & 4 & 5 & 6 & 7 & 8 & 9 & 10 \\
\hline Rumex thyrsiflorus & $\mathrm{H}$ & nd & sp? & MA & MA & 6510 & . & . & . \\
\hline Sagina nodosa & $\mathrm{C}, \mathrm{H}$ & $\mathrm{CB}(\mathrm{d})$ & $\mathrm{sp}$ & IJ & IJ, S & . & . & . & . \\
\hline Sagina procumbens & $\mathrm{C}, \mathrm{T}$ & $\mathrm{CB}(\mathrm{d})$ & $\mathrm{sp}$ & $\mathrm{PP}$ & PP & . & . & . & . \\
\hline Salix aurita & $\mathrm{N}$ & sa-CE & $\mathrm{sp}$ & $\mathrm{Ag}$ & $\mathrm{Ag}$ & . & . & . & . \\
\hline Salix caprea & $\mathrm{M}, \mathrm{N}$ & sa-ES-M(n)-IR(w) & $\mathrm{sp}$ & E & $\mathrm{E}$ & . & . & . & . \\
\hline Salix cinerea & $\mathrm{N}$ & $\mathrm{ES}(\mathrm{w})$ & $\mathrm{sp}$ & $\mathrm{Ag}$ & $\mathrm{Ag}$ & . & . & . & . \\
\hline Salix fragilis & M & sa-CE-M(n)-IR(w) & $\mathrm{sp}$ & $\mathrm{Sp}$ & $\mathrm{A} / \mathrm{MA}$ & ${ }^{*} 91 \mathrm{E} 0$ & . & . & . \\
\hline Salix pentandra & $\mathrm{M}, \mathrm{N}$ & ES & $\mathrm{sp}$ & $\mathrm{Ag}$ & $\mathrm{Ag}$ & . & . & . & . \\
\hline Sambucus nigra & $\mathrm{N}$ & sa-CE-M & $\mathrm{sp}$ & $\mathrm{RP}$ & QF, RP & . & . & . & . \\
\hline Scirpus sylvaticus & G & sa-ES(d) & $\mathrm{sp}$ & MA & MA & . & . & LC & . \\
\hline Scleranthus annuиs & $\mathrm{T}$ & W-med & ar & S & S & . & . & . & . \\
\hline Scrophularia nodosa & $\mathrm{H}$ & sa-ES(w) & $\mathrm{sp}$ & QF & $\mathrm{A}, \mathrm{MA}, \mathrm{QF}$ & . & an & . & . \\
\hline Scutellaria galericulata & $\mathrm{H}$ & $\mathrm{CB}$ & $\mathrm{sp}$ & $\mathrm{Ag}$ & $\mathrm{Ph}$ & . & . & . & . \\
\hline Sedum maximum & $\mathrm{G}, \mathrm{H}$ & sa-ES-M(n) & $\mathrm{sp}$ & TG & TG & . & . & . & . \\
\hline Senecio jacobaea & $\mathrm{H}$ & sa-ES(w)-IR(m) & $\mathrm{sp}$ & - & $\mathrm{A} / \mathrm{MA}$ & . & . & . & . \\
\hline Senecio vernalis & $\mathrm{H}, \mathrm{T}$ & Eur SE \& Asia W & $\mathrm{kn}$ & $\mathrm{KC}$ & $\mathrm{A} / \mathrm{MA}$ & . & . & . & . \\
\hline Senecio vulgaris & $\mathrm{H}, \mathrm{T}$ & med-atl & ar & S & S & . & . & . & . \\
\hline Setaria pumila & $\mathrm{T}$ & S-SE Asia & ar & S & S & . & . & . & . \\
\hline Solanum dulcamara & $\mathrm{N}, \mathrm{li}$ & sa-ES-M-IR & sp & $\mathrm{Ag}$ & $\mathrm{Ag}, \mathrm{Ph}$ & . & . & . & . \\
\hline Solidago gigantea & $\mathrm{G}, \mathrm{H}$ & $\mathrm{Am} \mathrm{N}$ & $\mathrm{kn}$ & $\mathrm{A}$ & A & . & . & . & . \\
\hline Solidago virgaurea & $\mathrm{H}$ & sa-ES & $\mathrm{sp}$ & $\mathrm{CU}$ & $\mathrm{A} / \mathrm{MA}$ & 4030 & an & . & . \\
\hline Sonchus arvensis & $\mathrm{G}, \mathrm{H}$ & sa-ES & $\mathrm{sp}$ & A & A, MA, S & . & . & . & . \\
\hline Sonchus oleraceus & $\mathrm{H}, \mathrm{T}$ & med-atl & ar & $S$ & S & . & . & . & . \\
\hline Sorbus aucuparia & $\mathrm{M}, \mathrm{N}$ & sa-CE & $\mathrm{sp}$ & - & $\mathrm{Q}, \mathrm{QF}$ & . & . & . & . \\
\hline Sparganium erectum & Hy & sa-ES(w)-M(n) & $\mathrm{sp}$ & $\mathrm{Ph}$ & $\mathrm{Ph}$ & . & . & LC & . \\
\hline Spergula arvensis & $\mathrm{T}$ & $\mathrm{W}$-med & ar & S & S & . & . & . & . \\
\hline Spirodela polyrhiza & Hy & cosmopolitan & $\mathrm{sp}$ & $\mathrm{L}$ & $\mathrm{L}$ & . & . & . & . \\
\hline Stachys sylvatica & $\mathrm{H}$ & sa-ES(w)-M(n)-IR(n) & $\mathrm{sp}$ & QF & $\mathrm{A}, \mathrm{QF}$ & ${ }^{*} 91 \mathrm{E} 0,91 \mathrm{~F} 0$ & an & . & . \\
\hline Stellaria graminea & $\mathrm{H}$ & sa-ES(w)-M(e, n)-IR(m) & $\mathrm{sp}$ & MA & MA & . & . & . & . \\
\hline Stellaria holostea & $\mathrm{C}$ & sa-ES(w)-M(n, e) & $\mathrm{sp}$ & QF & QF & 9160 & an & . & . \\
\hline Stellaria media & $\mathrm{T}, \mathrm{H}$ & cosmopolitan & $\mathrm{sp}$ & S & A, S & . & . & . & . \\
\hline Stellaria nemorum & $\mathrm{H}$ & CE-M(n) & $\mathrm{sp}$ & QF & $\mathrm{MC}, \mathrm{QF}$ & ${ }^{*} 91 \mathrm{E} 0,91 \mathrm{~F} 0$ & an & . & . \\
\hline Tanacetum vulgare & $\mathrm{H}$ & sa-ES & $\mathrm{sp}$ & $\mathrm{A}$ & A & . & . & . & . \\
\hline Taraxacum officinale & $\mathrm{H}$ & sa-ES(w)-M-IR & $\mathrm{sp}$ & MA & A, MA, S & . & . & . & . \\
\hline Teesdalea nudicaulis & $\mathrm{H}, \mathrm{T}$ & CE: sat & $\mathrm{sp}$ & KC & KC, S & . & . & . & . \\
\hline Thlaspi arvense & $\mathrm{T}, \mathrm{H}$ & C Asia & ar & S & S & . & . & . & . \\
\hline Thymus serpyllum & $\mathrm{C}$ & $\mathrm{CE}(\mathrm{n})$ & sp & $\mathrm{KC}$ & $\mathrm{KC}$ & . & . & . & . \\
\hline Tilia cordata & M & sa-ES(w) & $\mathrm{sp}$ & QF & QF & 9160 & an & . & . \\
\hline Torilis japonica & $\mathrm{H}, \mathrm{T}$ & sa-CE(e)-M-Asia ES(?) & $\mathrm{sp}$ & A & A & . & . & . & . \\
\hline Tragopogon pratensis & $\mathrm{H}$ & CE: ce-n & $\mathrm{sp}$ & MA & MA & 6510 & . & . & . \\
\hline Trifolium alpestre & $\mathrm{H}$ & $\mathrm{CE}-\mathrm{M}(\mathrm{n})$ & $\mathrm{sp}$ & TG & TG & . & . & LC & CWR \\
\hline Trifolium arvense & $\mathrm{T}$ & sa-ES(w)-M-IR(w) & sp & $\mathrm{KC}$ & $\mathrm{KC}$ & . & . & LC & CWR \\
\hline Trifolium campestre & $\mathrm{T}$ & sa-CE-M-IR & $\mathrm{sp}$ & $\mathrm{KC}$ & $\mathrm{KC}$ & . & . & . & . \\
\hline Trifolium medium & $\mathrm{H}$ & sa-ES(w) & $\mathrm{sp}$ & TG & TG & . & . & . & . \\
\hline Trifolium pratense & $\mathrm{H}$ & sa-ES-M-IR(m) & $\mathrm{sp}$ & MA & MA & . & . & LC & CWR \\
\hline
\end{tabular}


Tab. 1 (continued)

\begin{tabular}{|c|c|c|c|c|c|c|c|c|c|}
\hline 1 & 2 & 3 & 4 & 5 & 6 & 7 & 8 & 9 & 10 \\
\hline Trifolium repens & $\mathrm{C}, \mathrm{H}$ & sa-ES-M-IR & $\mathrm{sp}$ & MA & $\mathrm{A}, \mathrm{MA}, \mathrm{S}$ & . & . & $\mathrm{LC}$ & CWR \\
\hline Tussilago farfara & G & sa-ES(w)-M(n)-IR & $\mathrm{sp}$ & A & A & . & . & . & . \\
\hline Typha latifolia & $\mathrm{H}, \mathrm{Hy}$ & $\mathrm{CB}$ & $\mathrm{sp}$ & $\mathrm{Ph}$ & $\mathrm{Ph}$ & . & . & $\mathrm{LC}$ & . \\
\hline Urtica dioica & $\mathrm{H}$ & ES-M-IR-Asia E & $\mathrm{sp}$ & A & A, MA, QF & . & . & LC & . \\
\hline Vaccinium myrtillus & $\mathrm{Ch}$ & sa-ES & $\mathrm{sp}$ & $\mathrm{VP}$ & Q & . & an & . & . \\
\hline Veronica anagallis-aquatica & $\mathrm{H}$ & sa-ES-M-IR & $\mathrm{sp}$ & $\mathrm{Ph}$ & $\mathrm{Ph}$ & . & . & $\mathrm{LC}$ & . \\
\hline Veronica arvensis & $\mathrm{T}$ & nd & $\mathrm{sp} ?$ & S & S & . & . & . & . \\
\hline Veronica beccabunga & Hy, C & sa-ES(w)-M(n)-IR & $\mathrm{sp}$ & $\mathrm{MC}$ & MA & . & . & $\mathrm{LC}$ & . \\
\hline Veronica chamaedrys & $\mathrm{C}$ & sa-ES-M(n) & $\mathrm{sp}$ & MA & A, MA, QF & 6510 & . & . & . \\
\hline Veronica dillenii & $\mathrm{T}$ & $\mathrm{ES}(\mathrm{n})$ & $\mathrm{sp}$ & $\mathrm{KC}$ & $\mathrm{KC}$ & . & . & . & . \\
\hline Veronica hederifolia & $\mathrm{T}$ & sa-CE-M-IR(w) & $\mathrm{sp}$ & S & S & . & . & . & . \\
\hline Veronica officinalis & $\mathrm{C}$ & ES & $\mathrm{sp}$ & $\mathrm{CU}$ & Q & 4030 & . & . & . \\
\hline Veronica persica & $\mathrm{T}$ & Asia SW & $\mathrm{kn}$ & S & S & . & . & . & . \\
\hline Veronica serpyllifolia & $\mathrm{H}$ & sa-ES-M(n) & $\mathrm{sp}$ & MA & MA & . & . & . & . \\
\hline Veronica triphyllos & $\mathrm{T}$ & ar. resist & ar & S & $\mathrm{S}$ & . & . & . & . \\
\hline Viburnum opulus & $\mathrm{N}$ & sa-ES(w) & $\mathrm{sp}$ & $\mathrm{RP}$ & QF, RP & . & an & . & . \\
\hline Vicia angustifolia & $\mathrm{T}$ & sa-ES(w)-M-IR & $\mathrm{sp}$ & S & $\mathrm{S}$ & . & . & . & . \\
\hline Vicia cracca & $\mathrm{H}$ & sa-ES-M(n)-IR(m) & $\mathrm{sp}$ & MA & $\mathrm{A}, \mathrm{MA}$ & . & . & . & . \\
\hline Vicia hirsuta & $\mathrm{T}$ & med-atl & ar & S & S & . & . & . & . \\
\hline Viola arvensis & $\mathrm{T}$ & nd & ar & S & S & . & . & . & . \\
\hline Viola canina & $\mathrm{H}$ & sa-ES(w) & $\mathrm{sp}$ & $\mathrm{CU}$ & Q & 4030 & . & . & . \\
\hline Viola palustris & $\mathrm{H}$ & CB: c-b-o & $\mathrm{sp}$ & $\mathrm{SC}$ & $\mathrm{Ph}$ & 7140 & . & . & . \\
\hline Viola reichenbachiana & $\mathrm{H}$ & $\mathrm{CE}(\mathrm{w})-\mathrm{M}(\mathrm{n})$ & $\mathrm{sp}$ & QF & QF & . & an & . & . \\
\hline Viscum album & $\mathrm{pp}$ & sa-CE-M(n)-IR-Asia E & $\mathrm{sp}$ & - & $\mathrm{RP}$ & . & . & . & . \\
\hline
\end{tabular}

1. Name of species [27]; 2. Raunkiaer's life form [34]: C - herbaceous chamaephyte, G - geophyte, Hy - hydrophyte, H - hemicryptophyte, li - liana, M - megaphanerophyte, N - nanophanerophyte, pp - semiparasite, T - therophyte; 3. Geographic sub-element [28]: CB - CircumBoreal, CE - Europaean temperate, ES - Euro-Siberian, IR - Irano-Turanian, M - Mediterranean; origin of archaeophytes [30]: nd - no data; origin of kenophytes [31,32]: Am - America, Eur - Europe; 4. Geographical-historical status [29]: ar - archaeophyte, kn - kenophyte, sp - spontaneophyte; 5. Syntaxonomic rank [24,33]: A - Artemisietea, Ag - Alnetea glutinosae, B - Bidentetea tripartitae, CU - CallunoUlicetea, E - Epilobietea angustifoliae, FB - Festuco-Brometea, IJ - Isoëto-Juncetea, J - Juncetea maritimi, KC - Koelerio-Corynephoretea, L - Lemnetea, MA - Molinio-Arrhenatheretea, MC - Montio-Cardaminetea, Ph - Phragmitetea, Po - Potametea, PP - Polygono-Poetea, Q - Quercetea robori-petraeae, QF - Querco-Fagetea, RP - Rhamno-Prunetea, S - Stellarietea mediae, SC - Scheuchzerio-Caricetea, Sp Salicetea purpureae, TG - Trifolio-Geranietea, VP - Vaccinio-Piceetea; 6. Syntaxonomic rank of a phytocoenose occurring at the mapped locality of species, see explanations in the previous point 5; 7. Natura 2000 habitat code [25,26]: 3150 - natural eutrophic lakes (Nymphaeion, Potamion), 3260 - water courses (Ranunculion fluitantis), 4030 - dry heaths (Calluno-Genistion, Pohlio-Callunion, Calluno-Arctostaphylion), 6210 - semi-natural dry grasslands on calcareous substrates (Festuco-Brometea), 6410 - Molinia meadows, 6430 - hydrophilous tall herb fringe communities (Calystegion sepium), 6510 - lowland hay meadows (Arrhenatherion), 7140 - transition mires (Scheuchzerio-Caricetea), 9110 - Luzulo-Fagetum beech forests, 9130 - Galio odorati-Fagenion beech forests, 9160 - sub-atlantic oak-hornbeam forests (StellarioCarpinetum), ${ }^{*} 9180$ - Tilio-Acerion forests of slopes, 9190 - acidophilous oak forests (Quercion robori-petraeae); ${ }^{*} 91 \mathrm{E} 0$ - alluvial forests (Salicion albae, Fraxino-Alnetum), 91F0 - riparian mixed forests (Querco-Ulmetum), * - priority protection; 8. an - ancient woodland plant species indicator [39,40]; 9. Category of threat in countries of the European Union [38]: LC - least concern; 10. Crop wild relatives at risk of decline in countries of the European Union [38].

Holarctic element was dominated by Euro-Siberian (54), Circum-Boreal (51) and European - temperate (30) subelements. Among alien species (51), there were $36(11 \%)$ archaeophytes and $15(4 \%)$ kenophytes. Only 6 of 15 kenophytes found had their autecological optimum in arable lands and they showed only a very small contribution to weediness.
Five of them were among the most frequently occurring in the segetal flora of Poland. Most of the archaeophytes (31\%) were a Mediterranean element, while most of the kenophytes (47\%) came from areas of the Western Hemisphere. The analysis of the syntaxonomic spectrum indicated that the species were characteristic for 23 classes. The highest 
proportion of species was from Molinio-Arrhenatheretea (62), Stellarietea mediae (52), Artemisietea (45), QuercoFagetea (30), and Phragmitetea (19). Three partially protected taxa were found - Centaurium erythraea (N 53 ${ }^{\circ} 45^{\prime} 32.21^{\prime \prime} /$

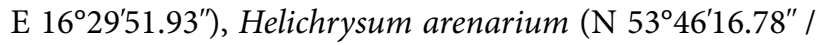
E 16 $29^{\prime} 51.24^{\prime \prime}$ ), and Platanthera bifolia (N 53 $46^{\prime} 12.98^{\prime \prime} /$ $\left.\mathrm{E} 16^{\circ} 29^{\prime} 45.54^{\prime \prime}\right)$. In the neighboring region of Wielkopolska, five species threatened with extinction were noted. These were Alchemilla monticola (N 534ㄴ $33.34^{\prime \prime} /$ E $16^{\circ} 29^{\prime} 52.61^{\prime \prime}$ ), Bromus secalinus (N 5345'32.12" / E 16²9'51.08",

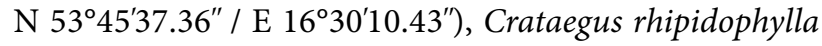

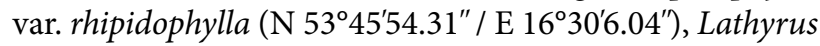
montanus (N 5346'21.39" / E 16²9'45.62'), and the aforementioned Platanthera bifolia. Also, 55 species endangered in the European Union territory were found, including 17 crop wild relatives at risk of decline. All of them belonged to native taxa. The vascular plant vegetation of Pustkowie was composed of phytoceoenoses (52 plant associations). They represented 17 classes, 23 orders and 32 alliances of the phytosociological system. Six segetal synanthropic associations from three alliances of Stellarietea mediae were identified: Panico-Setarion - Digitarietum ischaemi R. Tx. et Preising in R. Tx. 1950 ex R. Tx. 1954, Echinochloo-Setarietum pumilae Felföldy 1942 corr. Mucina 1993, Setario-Lycopsietum arvensis Pass. 1959, Spergulo arvensis-Scleranthetum annui Kuhn 1937, Spergulo-Echinochloetum cruris-galli (Kruseman et Vlieger 1939) R. Tx. 1950; Scleranthion annui - Papaveretum argemones Kruseman et Vlieger 1939, Sclerantho-Arnoseridetum minimae R. Tx. 1937; Veronico-Euphorbion - Veronico agrestis-Fumarietum officinalis R. Tx. in Lohmeyer 1949 ex J. Tx. 1955. A part of plants (72 spp. 21\%) were representative species for 15 Natura 2000 habitats. Only 6430 Convolvuletalia sepium, 6510 Arrhenatherion elatioris and 9160 Stellario-Carpinetum habitats had an adequate conservation status in the investigated area.

\section{Discussion}

The spontaneous vascular flora of Pustkowie, which is situated in the Drawsko Lakeland, was characterized by high plant species richness and plant diversity for such a relatively small area $-338 \mathrm{spp} . / 0.75 \mathrm{~km}^{2}$. The flora of other early glacial lakeland areas can serve as a point of reference for the interpretation of this result. $1039 \mathrm{spp} . / 900 \mathrm{~km}^{2}$ were reported from the Międzychód-Sieraków Lakeland [41], 1186 spp. / $2114 \mathrm{~km}^{2}$ from the Gniezno Lakeland [42], while $1120 \mathrm{spp} . /$ $51 \mathrm{~km}^{2}$ from the Wielkopolski National Park of the Poznan Lakeland [43]. The highest share of Asteraceae and Poaceae species in the flora of Pustkowie could result from the fact that numerous terophytes from these botanical families are characterized by somatic and physiological polymorphism of seeds [44]. Both features are important properties of the adaptation strategy in changing or unpredictable habitats and favor the formation of soil seed bank. The soil seed bank in agrophytocoenoses is particularly rich, 38 000-70 000 seeds / $1 \mathrm{~m}^{3}$ [44]. Probably, it was also abundant in the soils with lush synanthropic vegetation growing on all arable lands of Pustkowie. As many as 20 terophytes from Asteraceae and 9 from Poaceae occurred in either ruderal habitats from
Artemisietea or segetal habitats with Stellarietea mediae, or in both, while some of them (e.g., Digitaria ischaemum and Setaria pumila) formed facies. Magnoliophytina clearly dominated in Pustkowie (96\%) and their participation was almost the same (97\%) as in the flora of Poland [45]. Also, the pattern of Raunkiaer's spectrum was very similar. In the areas of Pustkowie, the aforementioned lakelands [41-43] and Poland [45], hemicryptophytes decidedly predominated over other life forms. The dominance of hemicryptophytes in floras has been recognized as a characteristic feature of the temperate climate zone [45].

The percentage of terophytes (28\%) in the flora of Pustkowie was higher than for Poland (20\%) [45] and this probably resulted from the local dominance of arable lands, their large habitat diversification and extensive cultivation. Private seed material, not purified from weed seeds, was used for several years. The soil was fertilized only with animal manure. Agrochemical agents were used sporadically and only in special situations (information from the land user). Thus, Pustkowie was a landscape unit used in conformity with the rules determined for organic farming [46]. It was reflected in the diversity of arable weeds and, among them, a substantial role of terophytes in the patches of six found segetal associations from the three alliances of Stellarietea mediae. In the phytocoenoses from Stellarietea mediae, 62 (67\%) terophytes occurred, while from Artemisietea - 30 (32\%). Phytocoenotic diversity was generated by the presence of slopes and depressions (high landform energy), generating numerous habitat katens. Habitat gradients and corresponding plant diversity (also phytocoenotic diversity) along the longitudinal axes of toposequences reflected, among others, the natural processes of water erosion. These processes were modified by agrotechnical denudation, enhanced by ploughing and sowing along the slopes, which formed lines of concentrated runoff of rainwater. Anthropogenic denudation, occurring within the slope katens of the Drawsko Lakeland agricultural lands, has been studied in details [47]. It has been shown that in arable lands water erosion proceeds in the upper and middle parts of a slope, while in its lower part and at the base there is an accumulation of washed material. This results in the systematic leveling of the surface irregularities in the lower part of the slope and in deepening the morphological contrast between the upper and lower parts. Thus, in future a different spatial distribution of segetal weeds and their communities can be expected in Pustkowie. In terms of the geosuccession rate, agricultural terraces were recognized as zones of the strongest and most dynamic transformations of landforms of the Drawsko Lakeland [47]. The flora modification will proceed not only as a consequence of water erosion, but presumably also as a result of significant changes in the chemical character of precipitation. Such changes were noted in the Geoecological Station UAM at Storkowo, situated only $1 \mathrm{~km}$ away from the northern border of Pustkowie.

In the hydrological year 2006, rain and snow water contained 6 ions: $\mathrm{NO}_{3}{ }^{-}-\mathrm{SO}_{4}{ }^{2-}-\mathrm{Cl}^{-}-\mathrm{NH}_{4}{ }^{+}-\mathrm{Na}^{+}-\mathrm{Ca}^{2+}$, while a year later - only 5 ions: $\mathrm{NO}_{3}{ }^{-}-\mathrm{Cl}^{-}-\mathrm{SO}_{4}{ }^{2-}-\mathrm{NH}_{4}{ }^{+}-\mathrm{Na}^{+}$. This was explained by the $\mathrm{SO}_{2}$ emission limits. In 2007 the acidity of precipitation reached a $\mathrm{pH}$ of 5.03 and this was the lowest value since 1994, similar to the water class with the so-called 
normal acidity. A distinct tendency for an increase in waters of such class was observed in the years 2002-2007. Since the end of the 1990s, the contribution of nitrogen oxides to the acidification of precipitation has been increasing. In 2007 the $\mathrm{NO}_{3}{ }^{-} / \mathrm{SO}_{4}{ }^{2-}$ indicator exceeded the value of 1 and therefore $\mathrm{NO}_{\times}$were responsible for acidification in a higher degree. Since the mid 1990s, precipitation decreased from ca. $8 \mathrm{t}$ $\mathrm{km}^{-2}$ to $3.3 \mathrm{t} \mathrm{km}^{-2}$ in 2006 [2]. In future, the spontaneous flora of vascular plants in Pustkowie, when mapped anew, will make it possible to determine the direction and scope of plant diversity transformations in this area as a result of changes in natural environment.

A balanced participation of spontaneophytes (49) and alien species (51, including 36 archaeophytes) among terophytes was observed. Some spontaneophytes (47 out of 287 , in majority terophytes) were a component of segetal coenoflora. The participation of natural species in agrocoenotic associations of Poland has been already reported [48-50]. Archaeophytes had a distinctly higher contribution to the composition of agrocoenoses than kenophytes. Only 3 out of 36 recorded archaeophytes did not occur in segetal vegetation. It was found [48] that the typical segetal communities of Poland are mostly based on archaeophytes and seem to be resistant to the invasion of alien species. This regularity was confirmed by the low participation of kenophytes in the segetal flora of Pustkowie. Only 6 kenophytes had an optimal distribution on arable lands. All of them contributed to weediness only in a small degree. They included: Veronica persica, Galinsoga parviflora, Oxalis fontana, Conyza canadensis, Chamomilla suaveolens, and Erigeron annuus. The first five made the sequence of kenophytes most frequently occurring in the segetal coenoflora of Poland [48]. Erigeron annuus was mainly noted in an orchard in sod and at the roadsides. In the catchment of the upper Parsęta, it was often observed as a pioneer species of early stages of secondary succession in abandoned croplands where it locally grew in abundance (own records). Also, the presence of Anthoxanthum aristatum was noted. This grass belongs to potentially invasive species, clearly increasing its range in the recent years [51]. Only 8 specimens occurred in one locality (N 53 $\left.{ }^{\circ} 45^{\prime} 51.34^{\prime \prime} / \mathrm{E} 16^{\circ} 29^{\prime} 58.67^{\prime \prime}\right)$ in Pustkowie, in Sclerantho-Arnoseridetum minimae, which may prove its recent arrival. An analysis of the coenoflora of Poland showed that the patches of the aforementioned association are the phytocoenotic center of occurrence of this grass [48]. The topogram for Anthoxanthum aristatum, included in the atlas of Polish flora, indicates that in the square ATPOL-BB $\left(10000 \mathrm{~km}^{2}\right)$, in which Pustkowie is located, this grass was found in only four squares of $100 \mathrm{~km}^{2}$ [52].

The dominant participation of spontaneophytes (85\%) in the flora of Pustkowie indicated the relatively low level of synanthropization, defined as the process of replacement of autochtonic with allochtonic components of flora [53]. Spontaneophytes played the most important role in the development of the floristic composition of associations of natural and semi-natural origin, determined in accordance with the criteria presented in the literature [54]. They formed phytocoenoses of 27 natural and 8 semi-natural associations from 14 classes of the syntaxonomic system. In terms of plant formations, these were the following phytocoenoses: forest
(Ag, Q, QF - explanation of abbreviations under Tab. 1), thicket (RP), clear-cutting (E), tall herb fringe communities - hygrophilous (A) and thermophilous (TG), aquatic and rushes (L, Po, MC, Ph), muddy terophytes (B), xeric sand calcareous grasslands (KC), meadows and pastures (MA), and carpet communities (MA, PP). They occurred in the patches or ecological corridors of Pustkowie. These are structural and functional elements of the landscape subject to anthropogenic fragmentation, distinguished in conformity with the concept of patch-matrix-corridor [55]. The saturation of the space with patches and corridors of a hydrographic network, dirt roads, balks and various linear ecotones was high and even within the matrix of arable lands (Fig. 1). Their natural and semi-natural vegetation was probably the source of spontaneophytes spreading to the segetal vegetation matrix. A peculiar structure was an orchard in sod, covering 13 ha in the northern part of Pustkowie. This type of fruit tree cultivation was observed repeatedly in the upper catchment of the Parsęta [6]. It is an effect of the change of arable lands of low soil quality class into, at first, mowed fallows and next into orchards with mowed sod. No traces of herbicide use were found. Spontaneous vegetation of the examined orchard was characterized by high plant diversity and it was composed mainly of native species of Artemisietea and Molinio-Arrhenatheretea (locally with Koelerio-Corynephoretea and Festuco-Brometea). Numerous sites in the orchard had five species of Poaceae from Molinio-Arrhenatheretea: Arrhenatherum elatior, Festuca rubra, Lolium perenne, Phleum pretense, and Poa pratensis. These are crop wild relatives, endangered in the European Union [38].

The northern part of the Pustkowie landscape underwent homogenization. The process of homogenization was defined as opposed to fragmentation [56]. Natural and semi-natural landscape structures merged functionally into a spatial whole. The semi-natural orchard vegetation (until recently a mowed fallow), in large part occupied by the Solidago virgaurea community (Molinio-Arrhenatheretea / Artemisietea), came into direct contact with forest vegetation (mainly Calamagrostio-Quercetum or Stellario-Carpinetum), surrounding the orchard from the west, north and east. The change of the former arable land into an orchard in sod is connected with a decrease in flora synanthropization and an increase in natural plant species richness. A similar direction of changes was observed as a result of secondary succession on the post-agricultural land in Białowieża [57] and in the Wielkopolski National Park [58]. During 36 years from the time when cultivation in Białowieża was abandoned and 25 years for the Wielkopolski NP, most anthropophytes were replaced with native species. An increase in species diversity of plants during early secondary succession stage on the former arable land was found, based on the research in five European countries [59]. The homogenization and decrease in flora synanthropization of the northern part of Pustkowie will probably favor the maintenance of old forest species, found in the patches of Stellario-Carpinetum and Calamagrostio-Quercetum, providing these patches will not be degraded by forest treatments and the outer forest border will continue to be the contact zone (ecotone zone) of natural and semi-natural vegetation. 


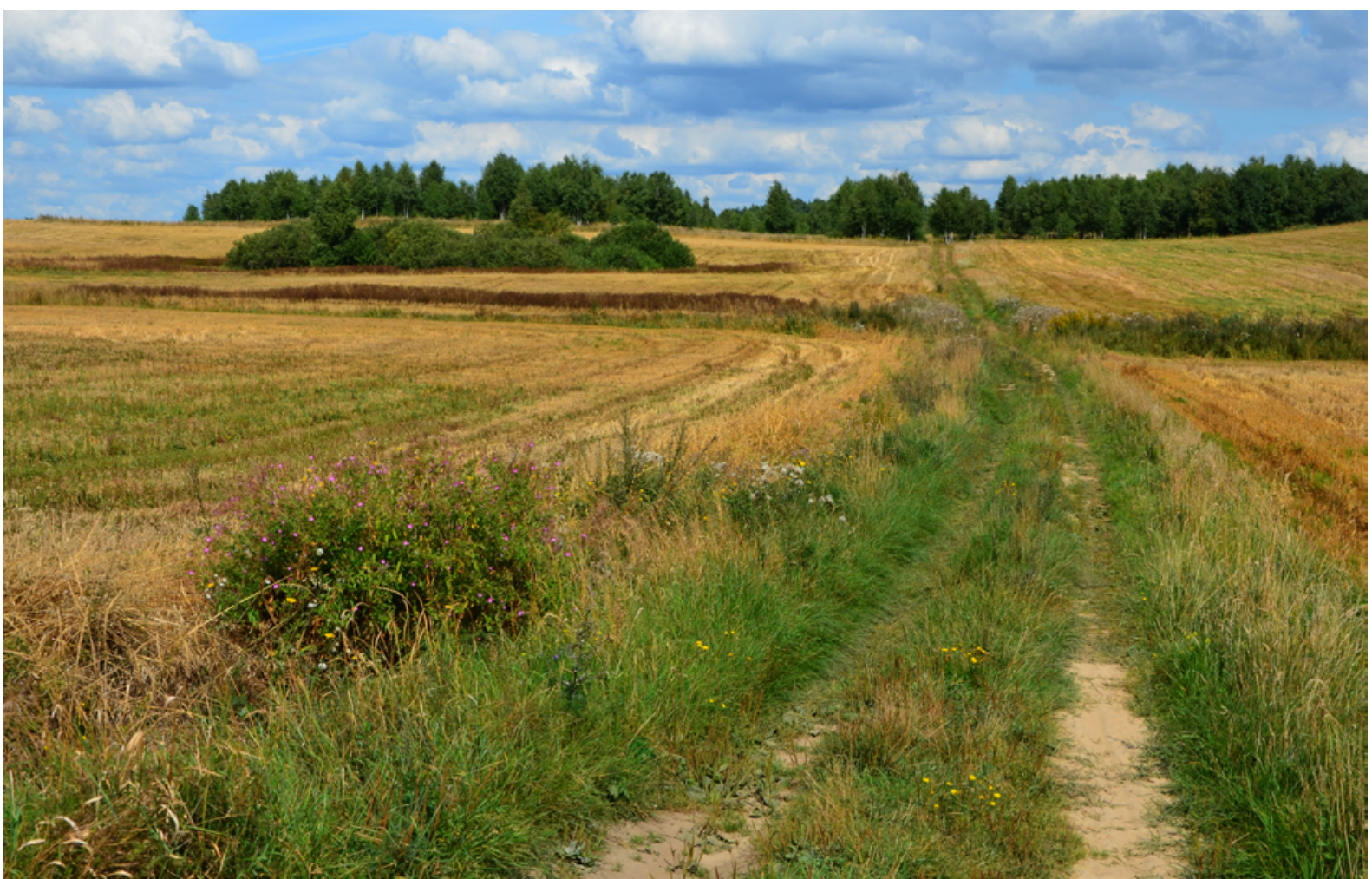

Fig. 1 Agricultural landscape with organic farms, patches and corridors of a hydrographic network, dirt roads, balks and various linear ecotones within the matrix of arable lands.

Presently, the spread of relic anthropophytes of the former farmland to these forests is limited by tall herb fringe vegetation developed in the ecotone zone between the orchard and the forest, serving as a natural ecological barrier. The preservation of old forest species is so important, because they are the indicator plants of natural habitats of Natura 2000: 6430, 9160 and 9190 [25], occurring in the area of Pustkowie (protected within PLH320007 Parsęta Catchment), adjacent to the orchard in sod from the north, east and south.

Thanks to extensive organic agriculture and the complex natural structure of the landscape, Pustkowie is characterized by a high level of plant diversity. A positive influence of ecological farming on biodiversity was documented [60]. It was found that extensification through organic management leads to greater biodiversity than in the case of higher-yielding conventional farming systems where the main structure in agricultural space comprises intensively managed annual crops. Such good biodiversity condition results, among others, from leaving natural and seminatural habitats in the landscape as well as weeds in crops. In Pustkowie these habitats occupied almost $40 \%$ of the analyzed area and weediness was exceptionally high. It was reported that leaving weeds in annual crops determines agroecosystem diversity, while biodiversity benefits of organic agriculture rely for a large part on non-crop plants growing within arable fields [14]. It was shown that plant species richness in organic farms was on average $34 \%$ higher than in conventional farms. This value differed depending on the taxonomic group and was significantly correlated with the proportion of arable fields [46].
In addition, the source of the high floristic diversity of Pustkowie was probably the location of farms, based on the known principles of location of prehistoric settlement sites in the Kaszuby Lakeland [61]. These principles involve the following: establishment of farms in the contact zones of different geocomplexes, within kames with lithological formations of finer fractions (aiding ploughing), with clay formations situated nearby ( $\leq 1 \mathrm{~km}$; for building purposes), close to biogenic accumulation plains with a relatively high level of groundwater (enabling meadow and pasture establishment), and at the same time in the proximity of available drinking water resources. Pustkowie has all the above-mentioned location features. The mosaic arrangement of its geocomplexes (among others, a depression with meadow and pasture, fragments of river valleys, 11 small water bodies, and midfield tree stands) provides numerous advantages, requires various ways of exploration, develops a multi-function economy and thus generates a high level of plant diversity.

The new EU and national government legislation, obligatory for farms entitled to direct subsidies, was introduced on January 1,2015 . This legislation is environmentally friendly and aims to support biodiversity. Among other things, it introduced payments for greening: diversification of cultivation for farms bigger than 10 ha of arable lands, conservation of environmentally valuable semi-natural grasslands in the areas of the Natura 2000 ecological network as well as for ecological focus areas for farms bigger than $15 \mathrm{ha}$. Ecological focus areas include, for example, small water bodies and peat bogs, drainage ditches ( $\leq 2 \mathrm{~m}$ wide), shrubs, tree-stand midfield patches, and balks. Due to this practice, the agricultural 
landscape of Pustkowie has a chance to preserve its unique plant species richness and diversity, as long as the user of its ecosystem services continues to practice organic farming and is entitled to direct subsidies [62].

The model object of the study was agricultural landscape situated about $2 \mathrm{~km}$ from an environmental monitoring station. This station has been recording, among others, the changes in natural environment, including climatic changes, for 30 years. This article documents the present condition of the spontaneous vascular flora of the studied landscape. Repeating such investigations in future will make it possible to determine the influence of changes in the environment on the richness and diversity of the flora, assuming that organic farming will be continued.

\section{Conclusions}

Extensive cultivation of arable lands and grasslands as well as keeping numerous field margins and fallows among crop fields were beneficial for species richness in the agricultural landscape.

The vascular flora of the extensively used agricultural landscape located in West Pomerania (75 ha) consisted of 338 species and was taxonomically diverse, comprising 213

\section{Acknowledgments}

I gratefully acknowledge the anonymous reviewers. Their valuable and useful comments led to many improvements in the paper. The research was supported by the Ministry of Science and Higher Education of Poland as part of the statutory activities of the Department of Integrated Geography, Adam Mickiewicz University in Poznań.

\section{Competing interests}

No competing interests have been declared.

\section{References}

1. Łowicki D, Mizgajski A. Typology of physical-geographical regions in Poland in line with land-cover structure and its changes in the years 1990-2006. Geogr Pol. 2013;86:255-266. http://dx.doi.org/10.7163/ GPol.2013.22

2. Kostrzewski A, Zwoliński Z, Andrzejewski L, Florek W, Mazurek M, Niewiarowski W, et al. Współczesna ewolucja rzeźby młodoglacjalnej Niżu Polskiego. In: Starkel L, Kotarba A, Kostrzewski A, Krzemień $\mathrm{K}$, editors. Współczesne przemiany rzeźby Polski. Kraków: IGiGP UJ; 2008. p. 271-325.

3. Piech J. Identyfikacja regionalnej specyfiki struktury obszarowej indywidualnych gospodarstw rolnych w Polsce. Zesz Nauk Akad Rol im H Kołłątaja Krak. 2001;20:107-116.

4. Babczyńska-Sendek B, Błońska A, Hejdysz J. Characteristic of the flora of fallow lands on rendzina soils on the Twardowice Plateau (Silesian Upland). Acta Agrobot. 2012;65(4):75-90. http://dx.doi. org/10.5586/aa.2012.024

5. Borysiak J, Mazurek M, Zwoliński Z. Concept of sustainable management involves landscape geodiversity of hydrogeomorphological units: the Dębnica River, Poland. In: The 8th IAG/AIG International conference on geomorphology and sustainability; 2013 Aug 27-31; Paris, France. Paris: International Association of Geomorphologists; 2013.

6. Borysiak J, Mazurek M, Zwoliński Z. Land cover and ecosystem services changes in agricultural landscapes of the Dębnica River catchment (West Pomerania, Poland). Ekonomia i Środowisko. 2014;4(51):205-214.

7. Robinson RA, Sutherland WJ. Post-war changes in arable farming and genera, 71 families and 48 orders, and was similar to other floras of lakeland areas of Poland.

The flora was dominated by spontaneophytes (83\%), which proves a low level of synanthropization. Spontaneophytes represented three main groups: connective (50\%), Holarctic (47\%), and cosmopolitan (3\%).

The study found a high richness of archaeophytes (11) which were composed of the segetal and ruderal flora; most of them represented a Mediterranean element. Very high weediness of arable lands prevented the migration of kenophytes; most of them (47\%) came from the areas of the Western Hemisphere.

The vascular flora was characterized by a highly diversified syntaxonomic spectrum - the species represented 23 classes of the phytosociological system. Molinio-Arrhenatheretea (62 species), Stellarietea mediae (52), and Artemisietea (45) had the highest participation.

The flora of the extensively used agricultural landscape is characterized by a high richness of spontaneous vascular flora and high taxonomic, syntaxonomic, geographic and life form spectra diversity. The study found the presence of 72 species representative for 15 types of Natura 2000 habitats, 45 forest ancient plant species, 55 species endangered in the European Union (including 17 crop wild relatives at risk of decline), and 5 regionally threatened species.

biodiversity in Great Britain. J Appl Ecol. 2002;39:157-176. http:// dx.doi.org/10.1046/j.1365-2664.2002.00695.x

8. Dembek W. Problemy ochrony polskiej przyrody w kontekście wspólnej polityki rolnej. Woda - Środowisko - Obszary Wiejskie. 2012;12(4):109-121.

9. Ministerstwo Rolnictwa i Rozwoju Wsi [Internet]. Program Rozwoju Obszarów Wiejskich (PROW) na lata 2014-2020. 2014 [cited 2015 Jan 10]; Available from: http://www.minrol.gov.pl/pol/ Wsparcie-rolnictwa-i-rybolowstwa/PROW-2014-2020

10. European Commission, Directorate-General for the Environment. Mapping and assessment of ecosystems and their services. Luxembourg: Publications office of the European Union; 2013. http://dx.doi. org/10.2779/12398

11. Council of Europe [Internet]. European Landscape Convention. 2000 [cited 2015 Jan 10]; Available from: http://conventions.coe.int/Treaty/ en/Treaties/Html/176.htm

12. Richling A, Solon J. Ekologia krajobrazu. Warszawa: Wydawnictwo Naukowe PWN; 2011.

13. European Commission [Internet]. EU Biodiversity Strategy to 2020. 2011 [cited 2015 Jan 10]; Available from: http://ec.europa.eu/environment/nature/biodiversity/comm2006/2020.htm

14. Clough Y, Kruess A, Tscharntke T. Local and landscape factors in differently managed arable fields affect the insect herbivore community of a non-crop plant species. J Appl Ecol. 2007;44:22-28. http://dx.doi. org/10.1111/j.1365-2664.2006.01239.x

15. Kondracki J. Geografia regionalna Polski. Warszawa: Wydawnictwo Naukowe PWN; 1998.

16. Karczewski A. Morfogeneza strefy marginalnej fazy pomorskiej na obszarze lobu Parsęty w vistulianie (Pomorze Środkowe). Poznań: Wydawnictwo Naukowe Uniwersytetu im. Adama Mickiewicza w Poznaniu; 1989.

17. Woś A. Zarys stosunków klimatycznych w rejonie górnego odcinka zlewni Parsęty. In: Kostrzewski A, editor. Zintegrowany monitoring środowiska przyrodniczego. Stacja Bazowa Storkowo. Warszawa: Biblioteka Monitoringu Środowiska; 1994. p. 79-96.

18. Popielski W. Szczegółowa mapa geologiczna Polski 1:50 000, arkusz 
159 Barwice (N-33-81-D). Warszawa: Państwowy Instytut Geologiczny; 2001.

19. Popielski W. Szczegółowa mapa geologiczna Polski 1:50 000, arkusz 160 Szczecinek (N-33-82-C). Warszawa: Państwowy Instytut Geologiczny; 2004.

20. Popielski W. Objaśnienia do Szczegółowej mapy geologicznej Polski 1:50 000. Arkusz Barwice (159). Warszawa: Państwowy Instytut Geologiczny; 2005.

21. Mocek A. Pokrywa glebowa zlewni górnej Parsęty. In: Kostrzewski A, editor. Geoekosystem obszarów nizinnych. Wrocław: Ossolineum; 1993. p. 137-146.

22. Dynowska I. Typy reżimów rzecznych w Polsce. Kraków: Uniwersytet Jagielloński; 1971. (Zeszyty Naukowe Uniwersytetu Jagiellońskiego. Prace Geograficzne; vol 28).

23. Faliński JB. Kartografia geobotaniczna. Część 1. Zagadnienia ogólne. Kartografia florystyczna i fitogeograficzna. Warszawa: Polskie Przedsiębiorstwo Wydawnictw Kartograficznych im. E. Romera; 1990.

24. Matuszkiewicz W. Przewodnik do oznaczania zbiorowisk roślinnych Polski. 3rd ed. Warszawa: Wydawnictwo Naukowe PWN; 2011.

25. Herbich J, editor. Poradniki ochrony siedlisk i gatunków Natura 2000 - podręcznik metodyczny. Tom 1-5. Warszawa: Ministerstwo Środowiska; 2004 [cited 2015 Jan 10]; Available from: http://natura2000.gdos.gov.pl/strona/nowy-element-3

26. Rozporządzenie Ministra Środowiska z dnia 9 sierpnia 2012 r. zmieniające rozporządzenie $\mathrm{w}$ sprawie siedlisk przyrodniczych oraz gatunków będących przedmiotem zainteresowania Wspólnoty, a także kryteriów wyboru obszarów kwalifikujących się do uznania lub wyznaczenia jako obszary Natura 2000. Dz. U. z 2012 r., poz. 1041.

27. Mirek Z, Piękoś-Mirkowa H, Zając A, Zając M, editors. Flowering plants and pteridophytes of Poland. A checklist. Kraków: W. Szafer Institute of Botany, Polish Academy of Sciences; 2002. (Biodiversity of Poland; vol 1).

28. Zając A, Zając M. Elementy geograficzne rodzimej flory Polski. Kraków: Pracownia Chorologii Komputerowej UJ; 2009.

29. Jackowiak B. Antropogeniczne przemiany flory roślin naczyniowych Poznania. Poznań: Wydawnictwo Naukowe Uniwersytetu im. Adama Mickiewicza w Poznaniu; 1990. (Biologia; vol 42)

30. Zając A. Pochodzenie archeofitów występujących w Polsce. Kraków: Uniwersytet Jagielloński; 1979. (Rozprawy Habilitacyjne; vol 29).

31. Zając A, Zając M, Tokarska-Guzik B. Kenophytes in the flora of Poland: list, status and origin. Phytocoenosis. 1998;10(9):107-116.

32. Tokarska-Guzik B. The establishment and spread of alien plant species (kenophytes) in the flora of Poland. Katowice: Wydawnictwo Uniwersytetu Ślaskiego; 2005. (Prace Naukowe Uniwersytetu Śląskiego w Katowicach; vol 2372).

33. Brzeg A, Wojterska M. Zespoły roślinne Wielkopolski, ich stan poznania i zagrożenie. In: Wojterska M, editor. Szata roślinna Wielkopolsk i Pojezierza Południowopomorskiego. Poznań: Bogucki Wydawnictwo Naukowe; 2001. p. 39-110.

34. Zarzycki K, Trzcińska-Tacik H, Różański W, Szeląg Z, Wołek J, Korzeniak U. Ecological indicator values of vascular plants of Poland. Kraków: W. Szafer Institute of Botany, Polish Academy of Sciences; 2002.

35. Rozporządzenie Ministra Środowiska z dnia 9 października 2014 r. w sprawie ochrony gatunkowej roślin. Dz. U. z 2014 r., poz. 1409.

36. Żukowski W, Jackowiak B. Lista roślin naczyniowych ginących i zagrożonych na Pomorzu Zachodnim i w Wielkopolsce. In: Żukowski W, Jackowiak B, editors. Ginące i zagrożone rośliny naczyniowe Pomorza Zachodniego i Wielkopolski. Poznań: Zakład Taksonomii Roślin Uniwersytetu im. Adama Mickiewicza w Poznaniu; 1995. p. 7-96. (Prace Zakładu Taksonomii Roślin Uniwersytetu im. Adama Mickiewicza w Poznaniu; vol 3).

37. Jackowiak B, Celka Z, Chmiel J, Latowski K, Żukowski W. Red list of vascular flora of Wielkopolska (Poland). Biodiv Res Conserv. 2007;5-8:95-127.

38. Bilz M, Kell SP, Maxted N, Lansdown RV. European red list of vascular plants. Luxembourg: Publications Office of the Europaean Union 2011. http://dx.doi.org/10.2779/8515

39. Hermy M, Honnay O, Firbank L, Grashof-Bokdam C, Lawesson JE. An ecological comparison between ancient and other forest plant species in Europe, and the implications for forest conservation. Biol Conserv. 1999;91:9-22. http://dx.doi.org/10.1016/S0006-3207(99)00045-2

40. Dzwonko Z, Loster S. Wskaźnikowe gatunki roślin starych lasów i ich znaczenie dla ochrony przyrody i kartografii roślinności. Warszawa: Instytut Geografii i Przestrzennego Zagospodarowania, Polska Akademia Nauk; 2001. (Prace Geograficzne; vol 178).

41. Wojterska M. Struktura krajobrazów roślinnych Pojezierza Międzychodzko-Sierakowskiego. Poznań: Bogucki Wydawnictwo Naukowe; 2003.

42. Chmiel J. Flora roślin naczyniowych wschodniej części Pojezierza Gnieźnieńskiego i jej antropogeniczne przeobrażenia w wieku XIX i XX. Poznań: Zakład Taksonomii Roślin Uniwersytetu im. Adama Mickiewicza w Poznaniu; 1993. (Prace Zakładu Taksonomii Roślin Uniwersytetu im. Adama Mickiewicza w Poznaniu; vol 1).

43. Żukowski W, Latowski K, Jackowiak B, Chmiel J. Rośliny naczyniowe Wielkopolskiego Parku Narodowego. Poznań: Zakład Taksonomii Roślin Uniwersytetu im. Adama Mickiewicza w Poznaniu; 1995. (Prace Zakładu Taksonomii Roślin Uniwersytetu im. Adama Mickiewicza w Poznaniu; vol 4).

44. Czarnecka B. Strategie adaptacyjne roślin a skład gatunkowy fitocenoz. Wiad Bot. 1997;41(3-4):33-42.

45. Jackowiak B. Flora roślin naczyniowych Wielkopolski w zarysie. In: Wojterska M, editor. Szata roślinna Wielkopolski i Pojezierza Południowopomorskiego. Poznań: Bogucki Wydawnictwo Naukowe; 2001. p. 25-38.

46. Tuck SL, Winqvist C, Mota F, Ahnström J, Turnbull LA, Bengtsson J. Land-use intensity and the effects of organic farming on biodiversity: a hierarchical meta-analysis. J Appl Ecol. 2014;51:746-755. http:// dx.doi.org/10.1111/1365-2664.12219

47. Szpikowski J. Wpływ denudacji antropogenicznej na zmiany pokrywy glebowej i morfologię stoków na obszarze młodoglacjalnym (zlewnia Perznicy, dorzecze Parsęty). In: Kostrzewski A, Kolander R, editors. Funkcjonowanie geoekosystemów Polski w warunkach zmian klimatu i różnokierunkowej antropopresji. Warszawa: Biblioteka Monitoringu Środowiska; 2005. p. 183-194.

48. Balcerkiewicz S, Pawlak G. Rola gatunków obcych w zbiorowiskach segetalnych Polski. Fragm Agron. 2010;27(2):19-30.

49. Balcerkiewicz S, Pawlak G. Spontaneofity w zbiorowiskach segetalnych Polski. Fragm Agron. 2010;27(3):7-19.

50. Dąbkowska T, Sygulska P. Variations in weed flora and the degree of its transformation in ecological and extensive conventional cereal crops in selected habitats of the Beskid Wyspowy Mountains. Acta Agrobot. 2013;66(2):123-136. http://dx.doi.org/10.5586/aa.2013.029

51. Tokarska-Guzik B, Dajdok Z, Zając M, Urbisz A, Danielewicz W. Identyfikacja i kategoryzacja roślin obcego pochodzenia jako podstawa działań praktycznych. Acta Bot Siles. 2011;6:23-53.

52. Zając A, Zając M, editors. Atlas rozmieszczenia roślin naczyniowych w Polsce. Kraków: Pracownia Chorologii Komputerowej Instytutu Botaniki UJ; 2001.

53. Faliński JB. Synantropizacja szaty roślinnej - próba określenia istoty procesu i głównych kierunków badań. Phytocoenosis. 1972;1(3):157-170.

54. Faliński JB. Zbiorowiska autogeniczne i antropogeniczne. Próba określenia i klasyfikacji. Dyskusje fitosocjologiczne (4). Ekol Pol. 1969;15(2):173-182.

55. Forman RTT. Some general principles of landscape and regional ecology. Landsc Ecol. 1995;10(3):133-142. http://dx.doi.org/10.1007/ BF00133027

56. Solon J. Ocena różnorodności krajobrazu na podstawie analizy struktury przestrzennej roślinności. Warszawa: Instytut Geografii i Przestrzennego Zagospodarowania, Polska Akademia Nauk; 2002. (Prace Geograficzne; vol 185). 
57. Adamowski W, Bomanowska A. Forest return on an abandoned field secondary succession under monitored conditions. Acta Univ Lodz Folia Biol Oecol. 2010;7:49-73.

58. Balcerkiewicz S, Pawlak G. Antropofity na tle dynamiki roślinności - studium na podstawie długoterminowego eksperymentu na powierzchni stałej. Acta Bot Siles. 2011;6:63-80.

59. Hedlund K, Santa Regina J, van der Putten WH, Lepš J, Diaz T, Korthals GW, et al. Plant species diversity, plant biomass and responses of the soil community on abandoned land across Europe: idiosyncracy or above-belowground time lags. Oikos. 2003;10:45-58. http://dx.doi. org/10.1034/j.1600-0706.2003.12511.x

60. Gibson RH, Pearce S, Morris RJ, Symondson WOO, Memmott J. Plant diversity and land use under organic and conventional agriculture: a whole-farm approach. J Appl Ecol. 2007;44:792-803. http://dx.doi. org/10.1111/j.1365-2664.2007.01292.x

61. Kittel P. Uwarunkowania środowiskowe lokalizacji osadnictwa pradziejowego na Pojezierzu Kaszubskim i w północnej części Borów Tucholskich. Łódź: Instytut Archeologii Uniwersytetu Łódzkiego; 2005. (Monografie Instytutu Archeologii Uniwersytetu Łódzkiego; vol 4).

62. Centrum Doradztwa Rolniczego w Brwinowie [Internet]. Zazielenienie - nowy element płatności bezpośrednich. 2014 [cited 2015 Oct 4]; Available from: http://www.cdr.gov.pl/aktualnoci/cdr-informuje/1524zazielenienie-nowy-element-platnosci-bezposrednich

\section{Znaczenie ekstensywnego, rolniczego użytkowania krajobrazu w kształtowaniu bioróżnorodności spontanicznej flory naczyniowej Pomorza Zachodniego}

\section{Streszczenie}

Badania wskazują na rolę ekstensywnych praktyk rolniczych w kształtowaniu różnorodności spontanicznej flory naczyniowej Pomorza Zachodniego. Do praktyk tych należą: nawożenie organiczne, brak stosowania herbicydów i pestycydów, wysiewanie własnego ziarna nie oczyszczonego w chwastów, dywersyfikacja upraw, a także pozostawienie w obrębie pól uprawnych licznych naturalnych struktur krajobrazowych. Modelową jednostką wybraną do badań był młodoglacjalny krajobraz rolniczy $\mathrm{o}$ areale 75 ha, skaładajacy się z gruntów ornych, trwałych użytków zielonych, sadu w darni, a także oczkek wodnych, miedz śródpolnych, rowów melioracyjnych, dróg gruntowych. Skartowana została spontaniczna flora naczyniowa tego krajobrazu. Przeanalizowano gatunkowy skład flory, zróżnicowanie taksonomiczne, syntaksonomiczną range roślin, udział elementu geograficznego i historyczno-geograficznego (w tym archeofitów i kenofitów), formę życiową Raunkiaer'a, a także udział roślin objętych ochrona gatunkowa, zagrożonych w regionie i krajach Unii Europejskiej, zagrożonych dzikich krewniaków roślin uprawnych oraz wskaźnikowych starych lasów. Spontaniczną florę naczyniową tworzyło 338 gatunków, zróżnicowanych taksonomicznie (213 rodzajów, 71 rodzin i 48 rzędów). Gatunki te wchodziły w skład 52 zespołów roślinnych z 17 klas, 23 rzędów i 32 związków systemu fitosocjologicznego. We florze dominowały spontaneofity (83\%). Tworzyły bogato zróżnicowane spectrum elementów geograficznych. Stwierdzono bogactwo archeofitów (11\%), występujących głównie w cenoflorach sześciu zanotowanych zespołów segetalnych. Udział kenofitów był niewielki, a ich występowanie prawdopodobnie było ograniczone wyjątkowo dużym zachwaszczeniem gruntów ornych, konkurencja archeofitów i spontaneofitów. Flora reprezentowała aż 9 form życiowych Raunkiaer'a. Udział terofitów był wyższy (28\%) niż podawany dla flory Polski (20\%). Florę naczyniową cechowało bogato zróżnicowane spectrum syntaksonomiczne; gatunki należały do taksonów charakterystycznych z 23 klas systemu fitosocjologicznego. Jednostką krajobrazową cechowała duża przyrodnicza wartość spontanicznej flory naczyniowej. 